Luiz Fernando Ybarra Martins de Oliveira

\title{
CORRELAÇÃO E CONCORDÂNCIA ENTRE OS ACHADOS DA TOMOGRAFIA DE COERÊNCIA ÓPTICA INTRACORONÁRIA E DA ANGIOTOMOGRAFIA COMPUTADORIZADA DE 320 DETECTORES NA AVALIAÇÃO DA ATEROSCLEROSE CORONARIANA
}

\begin{abstract}
Tese apresentada à Sociedade Beneficente Israelita Brasileira Albert Einstein, para obtenção do Título de Doutor em Ciências da Saúde.
\end{abstract}


Luiz Fernando Ybarra Martins de Oliveira

\section{CORRELAÇÃO E CONCORDÂNCIA ENTRE OS ACHADOS DA TOMOGRAFIA DE COERÊNCIA ÓPTICA INTRACORONÁRIA E DA ANGIOTOMOGRAFIA COMPUTADORIZADA DE 320 DETECTORES NA AVALIAÇÃO DA ATEROSCLEROSE CORONARIANA}

Tese apresentada à Sociedade Beneficente Israelita Brasileira Albert Einstein, para obtenção do Título de Doutor em Ciências da Saúde.

Orientador: Prof. Dr. Marco Antônio Perin Coorientador: Dr. Adriano Mendes Caixeta 
O48c

Oliveira, Luiz Fernando Ybarra Martins de

Correlação e concordância entre os achados da tomografia de coerência óptica intracoronária e da angiotomografia computadorizada de 320 detectores na avaliação da aterosclerose coronariana / Luiz Fernando Ybarra Martins de Oliveira. -- São Paulo, 2018.

xiii, 47 f., il.

Tese (Doutorado) - Sociedade Beneficente Israelita Brasileira Albert Einstein, Instituto de Ensino e Pesquisa Albert Einstein, Programa de Pós-Graduação em Ciências da Saúde.

Título em inglês: Diagnostic accuracy of 320-detector computed tomography using the CAD-RADS reporting system for characterizing coronary atherosclerotic plaques: comparison with intravascular optical coherence tomography.

1. Tomografia computadorizada por raios X. 2. Tomografia de coerência óptica. 3. Doença das coronárias. 4. Doença da artéria coronariana.

NLM - WN 206

Elaborada pelo Sistema Einstein Integrado de Bibliotecas 
SOCIEDADE BENEFICENTE ISRAELITA BRASILEIRA ALBERT EINSTEIN

Coordenador do Curso de Pós-Graduação: Prof. Dr. Luiz Vicente Rizzo 


\section{CORRELAÇÃO E CONCORDÂNCIA ENTRE OS ACHADOS DA TOMOGRAFIA DE COERÊNCIA ÓPTICA INTRACORONÁRIA E DA ANGIOTOMOGRAFIA COMPUTADORIZADA DE 320 DETECTORES NA AVALIAÇÃO DA ATEROSCLEROSE CORONARIANA}

Presidente da banca: Prof. Dr. Marco Antônio Perin

BANCA EXAMINADORA

Membros titulares:

Prof. Dr. Fernando Bacal

Prof. Dr. José Armando Mangione

Prof. Dr. Alexandre Antônio Cunha Abizaid

Membros suplentes:

Prof. Dr. Carlos Vicente Serrano Junior

Prof. Dr. Flavio Tarasoutchi

Data de Aprovação: 06 / 07 / 2018 


\section{Dedicatória}

À minha esposa Marina, pelo amor, carinho, suporte, amizade e dedicação.

Aos meus filhos Luiz Henrique e Luiz Felipe, as razões do meu viver, minhas maiores alegrias.

Ao meu pai Luiz Antônio e à minha mãe Solange, por todo carinho, educação e apoio irrestrito.

À minha irmã Simone, pela parceria desde sempre. 


\section{Agradecimentos}

Ao meu orientador Prof. Dr. Marco Antônio Perin, pela oportunidade de realizar esta tese e confiança desde o início.

Ao meu coorientador Dr. Adriano Mendes Caixeta, por me encorajar a perseguir uma das experiências mais ricas que vivenciei.

Ao Hospital Israelita Albert Einstein e à Comissão de PósGraduação da Sociedade Beneficente Israelita Albert Einstein, que subsidiam o ensino e a pesquisa de uma maneira única na realidade brasileira.

Ao Dr. Gilberto Szarf, pela parceria, amizade, conselhos e por estar sempre pronto e disposto a ajudar.

Ao Dr. Walther Ishikawa, pela prontidão e ajuda na analise dos dados.

Ao Dr. Daniel Silva Chamié de Queiroz, por aceitar participar deste desafio.

À Bárbara e ao Edu, por todo suporte e ajuda na logística da captação dos dados e pelo companheirismo.

Ao meu grande amigo Eduardo Fiorentino Alves de Araújo e a todos os amigos do E68, parceiros de todos os momentos.

À equipe de Cardiologia Intervencionista da McGill University Health Centre, e em especial ao Dr. Stéphane Rinfret, por todos seus ensinamentos, e ao Dr. Nicolo Piazza, pela amizade e camaradagem.

Ao Prof. Dr. Antônio Carlos de Camargo Carvalho, pelo suporte e por sempre deixar as portas abertas.

Ao Justo e à Ciça, pelos conselhos de vida e acadêmicos.

Aos meus filhos, pela compreensão e pelo amor incondicional.

À Má, pela capacidade de amar e cuidar sem limites, por ser o alicerce, por me tornar uma pessoa melhor. 
Agradeço à Fundação de Amparo à Pesquisa do Estado de São Paulo pelo apoio financeiro para realização deste estudo (Processos números 2015/00485-0 e 2015/02239-6). 


\section{Sumário}

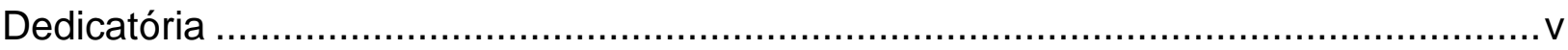

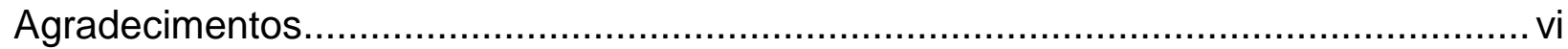

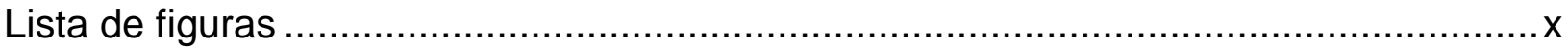

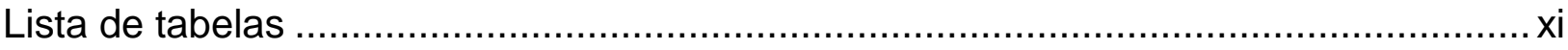

Lista de abreviaturas e símbolos ............................................................................ xii

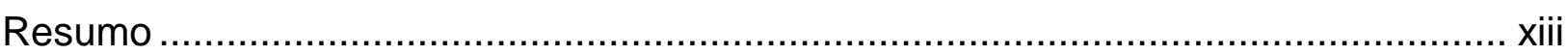

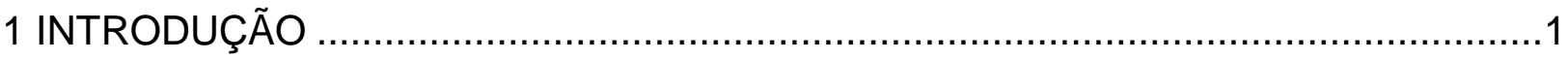

1.1 Aterosclerose

1.1.1 Estrutura da parede arterial .....................................................................

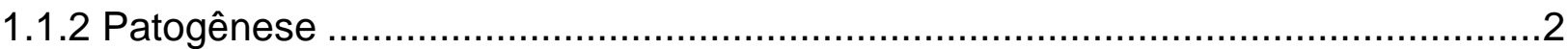

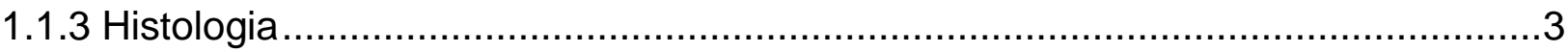

1.1.4 Ruptura e erosão da placa aterosclerótica ................................................4

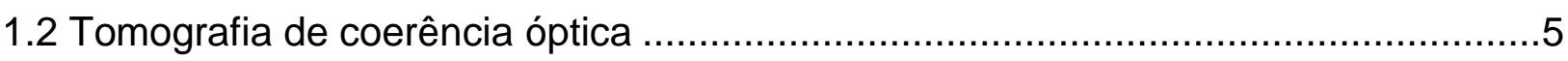

1.2.1 Tecnologia da tomografia de coerência óptica ..........................................5

1.2.2 Técnica e segurança da tomografia de coerência óptica.................................6

1.2.3 Imagens da tomografia de coerência óptica .................................................. 7

1.3 Angiotomografia computadorizada coronária .............................................. 8

1.3.1 Tecnologia da angiotomografia computadorizada coronária .............................8

1.3.2 Técnica e segurança da angiotomografia computadorizada coronária .................9

1.3.3 Imagens da angiotomografia computadorizada coronária ..............................10

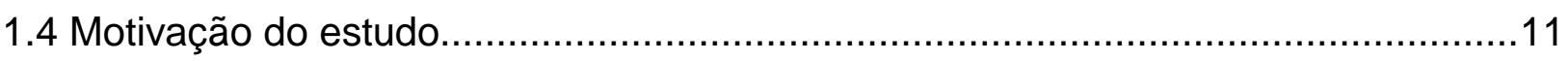

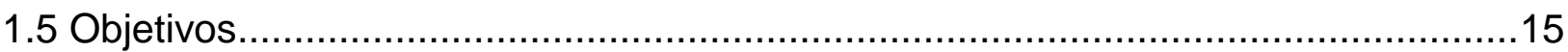

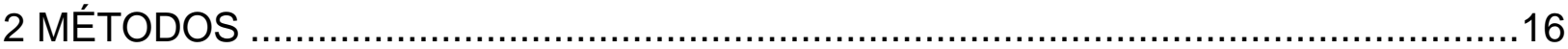

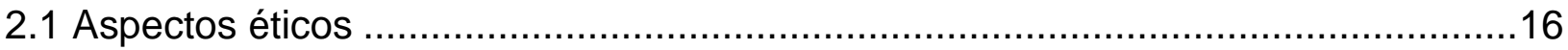

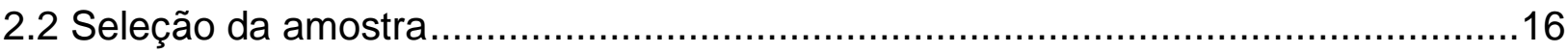

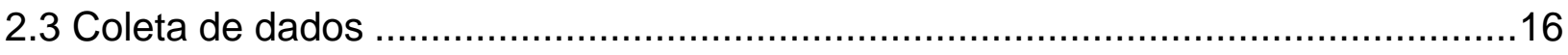

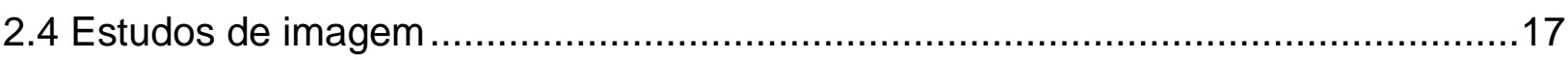

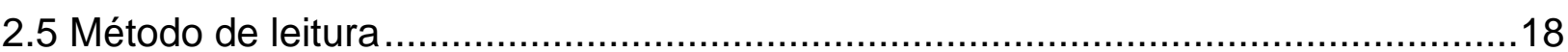

2.5.1 Coregistro das imagens....................................................................... 18

2.5.2 Leitura das imagens ................................................................................

2.5.3 Parâmetros quantitativos e qualitativos ......................................................19

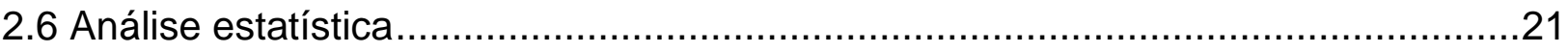

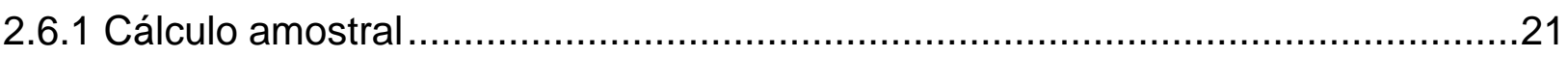

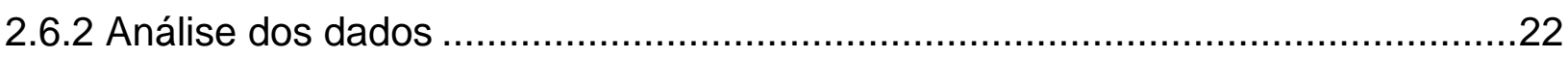




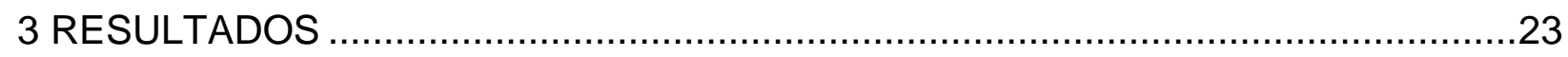

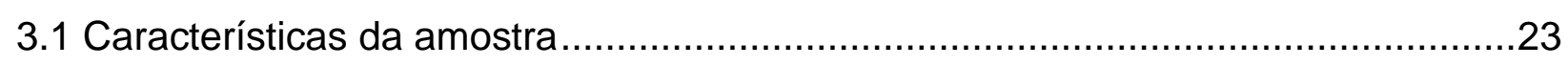

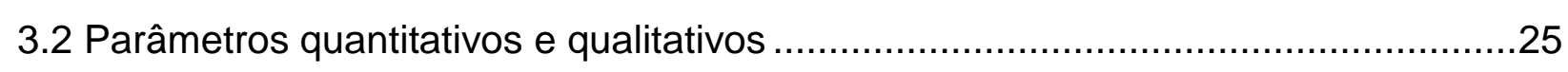

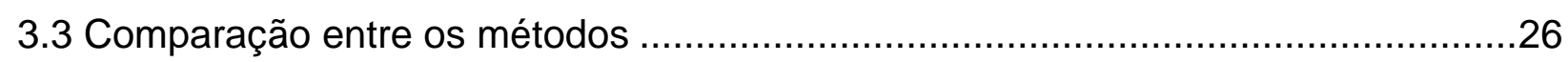

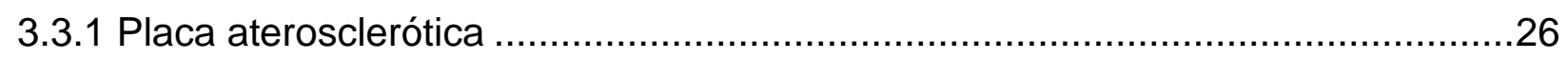

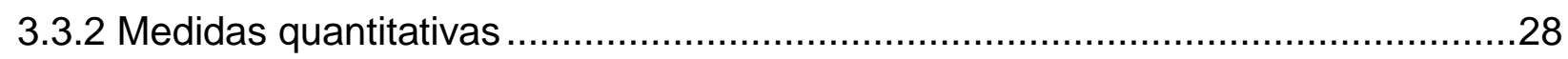

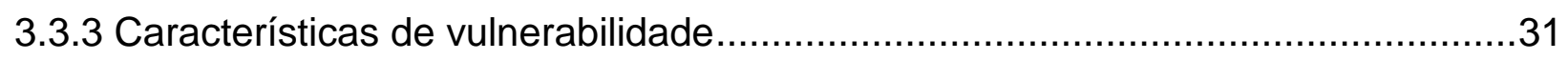

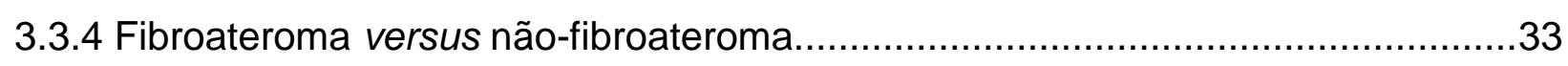

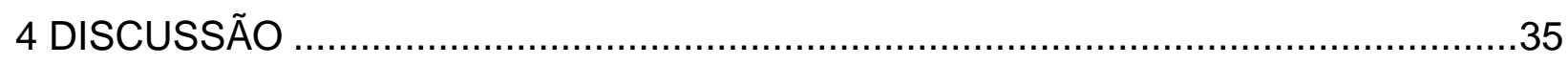

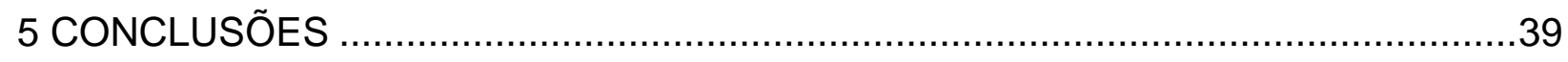

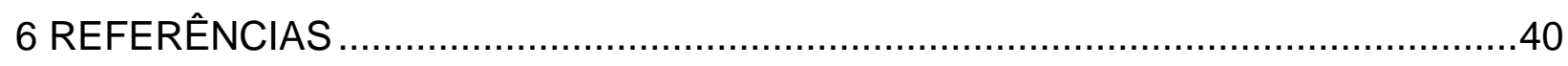

Abstract 


\section{Lista de figuras}

Figura 1. Representação das características tomográficas de vulnerabilidade 11

Figura 2. Modelo de coregistro: o segmento obtido na tomografia de coerência óptica era delimitado na cinecoronariografia e esta era utilizada para definir o segmento na tomografia computadorizada de 320 canais .................................................19

Figura 3. Fluxograma de distribuição da amostra ..........................................24

Figura 4. Diagramas de dispersão das medidas de área e de diâmetros entre as imagens obtidas pela tomografia de coerência óptica e pela tomografia computadorizada de 320 detectores 30

Figura 5. Gráficos de Bland-Altman das médias de diferenças e desvios padrões de área e de diâmetros entre as imagens obtidas pela tomografia de coerência óptica e pela tomografia computadorizada de 320 detectores 


\section{Lista de tabelas}

Tabela 1. Perfil clínico e demográfico dos pacientes da amostra .25

Tabela 2. Variáveis quantitativas e qualitativas avaliadas pela tomografia de coerência óptica e pela tomografia computadorizada de 320 detectores 26

Tabela 3. Descrição fenotípica da placa aterosclerótica observada nas imagens obtidas pela tomografia de coerência óptica e pela tomografia computadorizada de 320 detectores.

Tabela 4. Descrição fenotípica da classificação das placas ateroscleróticas em relação à classificação das placas ateroscleróticas calcificadas e não calcificadas observadas nas imagens obtidas pela tomografia computadorizada de 320 detectores

Tabela 5. Descrição de fibroateroma pela tomografia de coerência óptica e placa aterosclerótica não calcificada pela angiotomografia computadorizada de 320 detectores.

Tabela 6. Classificação das estenoses de acordo com a classificação pelo sistema CAD-RADS. .28

Tabela 7. Concordância das medidas quantitativas entre as imagens obtidas pela tomografia de coerência óptica e pela tomografia computadorizada de 320 detectores

Tabela 8. Medidas de acurácia das características de vulnerabilidade das imagens obtidas pela tomografia computadorizada de 320 detectores para a identificação de fibroateromas. 32

Tabela 9. Associação de sinais de vulnerabilidade da placa aterosclerótica das imagens obtidas pela tomografia computadorizada de 320 detectores e características de vulnerabilidade das imagens obtidas pela tomografia de coerência óptica . .33

Tabela 10. Associação das variáveis observadas à tomografia computadorizada de 320 detectores e à tomografia de coerência óptica com o fibroateroma definido pela tomografia de coerência óptica. .34 


\section{Lista de abreviaturas e símbolos}

\begin{tabular}{|c|c|}
\hline AngioTC & Angiotomografia computadorizada coronária \\
\hline bpm & Batimentos por minuto \\
\hline CAD-RADS & Coronary Artery Disease Reporting and Data System \\
\hline $\mathrm{cm}$ & Centímetro \\
\hline DP & Desvio padrão \\
\hline $\mathrm{HU}$ & Houndsfield units \\
\hline IAM & Infarto agudo do miocárdio \\
\hline IC95\% & Intervalo de confiança de $95 \%$ \\
\hline IMC & Índice de massa corpórea \\
\hline $\mathrm{Kg}$ & Quilograma \\
\hline $\mathrm{L}$ & Litro \\
\hline LDL & Colesterol de baixa densidade lipoproteica \\
\hline$m^{2}$ & Metros quadrados \\
\hline $\mathrm{mL}$ & Mililitros \\
\hline $\mathrm{mm}^{2}$ & Milímetros quadrados \\
\hline $\mathrm{mm}$ & Milímetro \\
\hline mseg & Milisegundo \\
\hline OCT & Tomografia de coerência óptica \\
\hline $\mathrm{p}$ & Valor-p \\
\hline PCR & Proteína $\mathrm{C}$ reativa de alta sensibilidade \\
\hline SCA & Síndrome coronariana aguda \\
\hline seg & Segundos \\
\hline TCDFRX & Tomografia computadorizada de dupla fonte de raio $X$ \\
\hline TCMD & Tomografia computadorizada com múltiplos detectores \\
\hline TC320 & Tomografia computadorizada de 320 detectores \\
\hline$\mu \mathrm{m}$ & Micrômetro \\
\hline
\end{tabular}




\section{Resumo}

Introdução: A angiotomografia computadorizada de múltiplos detectores é um método não-invasivo para caracterização da placa aterosclerótica coronária. Até o momento, nenhum estudo avaliou a capacidade da tomografia computadorizada de 320 detectores em determinar os diversos tipos de placa. Objetivo: Caracterizar a acurácia diagnóstica da tomografia computadorizada de 320 detectores em diferenciar as placas ateroscleróticas identificadas pela tomografia de coerência óptica. Métodos: Estudo transversal retrospectivo unicêntrico que avaliou imagens da tomografia computadorizada de 320 detectores e da tomografia de coerência óptica de 42 segmentos coronários de 32 pacientes. As imagens tomográficas foram interpretadas de acordo com a nova classificação CAD-RADS. As imagens da tomografia computadorizada de 320 detectores foram avaliadas em conjunto por dois radiologistas experientes e as da tomografia de coerência óptica, por um Corelab independente. Resultados: Observamos associação entre os achados das placas ateroscleróticas observados na tomografia de coerência óptica e aqueles observados pela tomografia computadorizada de 320 detectores quando estas foram classificadas como calcificadas e não calcificadas pela tomografia computadorizada de 320 detectores $(p=0,005)$, não havendo associação destas com a identificação de fibroateroma $(p=0,49)$. As concordâncias entre as medidas de diâmetros $(0,11$ a 0,20$)$ e área $(0,52)$ luminais dos dois métodos foram baixas, sendo que a tomografia computadorizada de 320 detectores subestima os valores de diâmetros luminais. A associação das características tomográficas de sinal do anel de guardanapo, remodelamento positivo, placas ateroscleróticas com baixa atenuação e calcificação localizada proporcionou a maior acurácia $(75,14 \%$, intervalo de confiança de $95 \% 60,8 ; 89,5)$. A presença de pelo menos duas destas características se associou com a presença de macrófagos e cristais de colesterol observados na tomografia de coerência óptica $(74,2 \%$ versus $25,8 \% ; p=0,034$ e $85,7 \%$ versus $14,3 \% ; p=0,040$, respectivamente). Houve uma tendência de maior presença de sinal do anel de guardanapo ( $80,0 \%$ versus $43,8 \%$, $\mathrm{p}=0,071$ ) nos fibroateromas observados pela tomografia de coerência óptica.

Conclusão: A tomografia computadorizada de 320 detectores foi capaz de diferenciar as placas ateroscleróticas calcificadas das não calcificadas identificadas pela tomografia de coerência óptica, contudo não conseguiu identificar os fibroateromas 


\section{INTRODUÇÃO}

A aterosclerose é um processo patológico cuja consequência é o desenvolvimento de doença coronária, cerebral, de artérias periféricas e da aorta. ${ }^{(1,2)}$ Sua evolução é geralmente assintomática até o momento em que a placa gere uma estenose que exceda 70 ou $80 \%$ do diâmetro luminal. Esta lesão estenótica pode produzir redução no fluxo sanguíneo, como no fluxo coronário para o miocárdio.

A cinecoronariografia é um método diagnóstico capaz de avaliar o lúmen das artérias coronárias, no entanto pode não ser suficiente para a avaliação das placas ateroscleróticas moderadamente estenóticas. ${ }^{(3,4)}$ Ela também não é capaz de caracterizar os diferentes tipos de placa aterosclerótica, que poderiam levar a distintas apresentações clínicas da doença coronariana, como, por exemplo, a síndrome coronariana aguda (SCA) da angina estável. ${ }^{(5-7)}$

Sendo assim, a avaliação da placa aterosclerótica por meio de outras técnicas invasivas como o ultrassom intravascular e a tomografia de coerência óptica (OCT), assim como técnicas de imagem não-invasivas como ressonância nuclear magnética e a tomografia computadorizada com múltiplos detectores (TCMD) estão ganhando espaço e importância. ${ }^{(8)}$

\subsection{Aterosclerose}

A palavra aterosclerose tem origem grega e significa acumulo endurecido focal de lipídeo. A aterosclerose pode começar já na adolescência e progride com o envelhecimento. ${ }^{(9-11)}$ Embora seja uma doença sistêmica, ela se manifesta de maneira focal e pode afetar diferentes órgãos em cada paciente por razões ainda não esclarecidas.

\subsubsection{Estrutura da parede arterial}

A fim de entender o processo de aterosclerose, assim como os métodos de imagem, devemos primeiramente nos familiarizar com a estrutura normal da parede arterial 
A intima é a camada mais interna que está em contato direto com o espaço intra-luminal. Sua espessura é de apenas uma ou duas camadas de células, mas pode se espessar muito mais com a formação e o crescimento da placa aterosclerótica. Ela se separa da camada média pela membrana elástica interna.

A média é a camada do intermediária da parede arterial e é composta predominantemente de camadas homogêneas de células de musculo liso, que regulam o tônus vascular. Separa-se da camada adventícia pela membrana elástica externa. A camada adventícia circunda a camada média e é composta por tecido conectivo fibrótico, fornecendo um suporte externo ao vaso.

\subsubsection{Patogênese}

Múltiplos fatores contribuem para a patogênese da aterosclerose, dentre eles: disfunção endotelial, dislipidemia, fatores imunológicos e inflamatórios, ruptura da placa aterosclerótica e tabagismo.

Disfunção na vasodilatação endotelial é o passo inicial na aterosclerose e acredita-se que ocorra principalmente pela perda do oxido nítrico proveniente do endotélio. ${ }^{(12)} \mathrm{O}$ endotélio vascular possui uma interface biológica ativa entre o sangue e os demais tecidos. A camada simples de endotélio continuo dentro das artérias forma uma camada trombo-resistente única entre o sangue e tecidos subendoteliais potencialmente trombogênicos. $O$ endotélio também modula 0 tônus, o crescimento, a hemostasia e a inflamação no sistema circulatório.

A dislipidemia tem um papel fundamental no desenvolvimento da aterosclerose. Níveis elevados de colesterol de baixa densidade lipoproteica (LDL) são um fator de risco importante para aterosclerose. ${ }^{(13-15)} \mathrm{O} L D L$ se acumula nos macrófagos cheios de lipídeos (células espumosas) e no núcleo lipídico da placa aterosclerótica. ${ }^{(16)} \mathrm{O}$ acumulo de LDL nas células espumosas levam a disfunção mitocondrial, apoptose e necrose, resultando em liberação de proteases celulares, citocinas inflamatórias e moléculas pró-trombóticas. ${ }^{(17)}$

A inflamação também exerce um papel central no entendimento da patogênese da aterosclerose. Indícios de inflamação em lesões ateroscleróticas foram observados nos primeiros estudos histológicos de placas ateroscleróticas. ${ }^{(18,19)}$ Recentemente, o estudo CANTOS demonstrou que, em pacientes com infarto do 
miocárdio prévio, a inibição da interleucina-1 beta com 0 anti-inflamatório canakinumab subcutâneo reduziu substancialmente os biomarcadores inflamatórios proteína $C$ reativa de alta sensibilidade (PCR) e interleucina- 6 sem mudar os lipídeos aterogênicos. O risco de um desfecho composto de morte cardiovascular, infarto do miocárdio não-fatal e acidente vascular cerebral não-fatal caiu $15 \%(p=0,021)$ com o uso de canakinumab $150 \mathrm{mg}$ subcutâneo a cada 3 meses.

\subsubsection{Histologia}

Histologicamente, a primeira fase da aterosclerose ocorre com o espessamento focal da intima com o acumulo de macrófagos cheios de lipídeos (células espumosas) e matriz extracelular. ${ }^{(20)}$ As células espumosas constituem o marco do começo do ateroma. Células de musculo liso também podem estar presentes na intima, surgindo das células-tronco hematopoiéticas. ${ }^{(21)}$ Os lipídeos acumulam-se precocemente na formação das estrias gordurosas, resultando em depósitos de lipídeos intra e extracelular.

À medida que estas lesões se expandem, mais células de músculo liso se acumulam na íntima. As células do músculo liso dentro da camada profunda das estrias gordurosas podem sofrer apoptose, o que se associa a mais acúmulo de macrófagos e microvesículas. Estas podem calcificar, contribuindo para a transição de estrias gordurosas em placas ateroscleróticas. ${ }^{(22)}$

Ateromas com capa fibrosa são placas ateroscleróticas com um núcleo lipídico bem definido recoberto por uma capa fibrótica na porção luminal, seja ela relativamente acelular (densa em colágeno) ou rica em células lisas.

Os vasa vasorum formam uma rede de microvasos que se originam da camada adventícia de grandes artérias. Estes microvasos fornecem oxigênio e nutrientes para as camadas mais externas da parede arterial. ${ }^{(23)}$ À medida que as placas ateroscleróticas se desenvolvem e expandem, elas adquirem suas próprias redes microvasculares, que se estendem da adventícia, através da mídia, até a intima espessada. ${ }^{(24)}$ Esses microvasos de paredes finas são susceptíveis a ruptura, levando a hemorragia interna da placa aterosclerótica e contribuindo com a progressão da aterosclerose coronária. ${ }^{(25)}$ Lesões mais avançadas contêm geralmente um núcleo necrótico rico em lipídeos e, ocasionalmente, regiões 
localizadas com calcificação. ${ }^{(9)}$

A formação do ateroma se associa com remodelamento da artéria coronária. ${ }^{(26)} \mathrm{O}$ remodelamento arterial é associado a uma fisiologia arterial anormal e pode estar associado ao desenvolvimento de sintomas $_{\text {clínicos. }}{ }^{(26)} \mathrm{O}$ remodelamento positivo se refere ao local de maior área da membrana elástica externa que ocorre de forma compensatória à expansão do tamanho do vaso devido a um aumento da placa aterosclerótica. Ele atua como um mecanismo compensatório nos primeiros estágios da doença arterial coronária, prevenindo uma perda luminal apesar do progressivo acúmulo de placa aterosclerótica, e pode ser observado em placas ateroscleróticas complexas instáveis em pacientes com angina instável. O remodelamento negativo se refere à menor área da membrana elástica externa no local da lesão em razão de uma diminuição do tamanho do vaso e costuma ser associado a placas ateroscleróticas obstrutivas em pacientes com angina estável. ${ }^{(27)}$

\subsubsection{Ruptura e erosão da placa aterosclerótica}

A progressão das placas ateroscleróticas envolve dois processos distintos: um crônico, que leva a estreitamento luminal lento, e um agudo, que causa uma rápida obstrução luminal associado a hemorragia na placa aterosclerótica e/ou trombose luminal.

A SCA ocorre tipicamente como resultado de uma ruptura ou erosão da placa aterosclerótica, resultando em trombose coronária. Estas placas ateroscleróticas podem ter estenoses menores de $50 \% .{ }^{(28,29)}$ No local da erosão não é possível observar endotélio e a intima exposta é composta predominantemente de células de musculo liso e proteoglicanos. Estudos clínicos com OCT realizados em pacientes com infarto agudo do miocárdio (IAM) mostraram uma incidência de ruptura da capa fibrosa de $73 \%$, enquanto que erosão da placa aterosclerótica estava presente em $83 \%$, resultados muito semelhantes à estudos em autopsias. ${ }^{(30)}$

Ruptura ou erosão da placa aterosclerótica podem ser um evento silencioso. Repetidas rupturas com trombose silenciosas seguidas de cicatrização das lesões, podem levar a progressão da aterosclerose, e estão associadas a um aumento da carga de placa aterosclerótica, do percentual de estenose e do remodelamento arterial negativo. ${ }^{(31)}$ 


\subsection{Tomografia de coerência óptica}

A cinecoronariografia é o método padrão-ouro para diagnóstico da extensão e da gravidade da estenose provocada pela placa aterosclerótica na circulação coronária. A necessidade de revascularização é, com frequência, baseada nas informações obtidas neste procedimento, assim como em dados clínicos e métodos não-invasivos. No entanto, a cinecoronariografia está sujeita ao viés de interpretação do operador e à variabilidade interoperador, além de também apresentar limitações técnicas, como não possibilitar a visualização de lesões especificas e somente ser capaz de visualizar características do contorno do lúmen vascular e não dos componentes da parede do vaso.

Com uma resolução 10 vezes superior ao ultrassom intravascular, a OCT é capaz de superar estas limitações. A OCT utiliza a reflexão do comprimento da onda de luz para obter imagens transversais do vaso, permitindo visualizar as coronárias com grande nível de detalhamento e fornecendo compreensão única da fisiopatologia do vaso.

Estas vantagens dão à OCT uma condição de instrumento de pesquisa reconhecido para a avaliação da aterosclerose. As indicações para o uso da OCT são semelhantes àquelas estabelecidas para o ultrassom intravascular e também podem ser usadas no processo de decisão clinico em pacientes com $\operatorname{SCA} .^{(32,33)}$

\subsubsection{Tecnologia da tomografia de coerência óptica}

A física por trás da OCT é análoga àquela da imagem de ultrassom, mas, ao invés de som, a luz é usada para obtenção das imagens. ${ }^{(34)}$ Tanto a largura de banda da luz infravermelha usada quanto a velocidade da onda são de ordens de magnitude mais altas do que as utilizadas na ultrassonografia médica. A resolução axial resultante acaba sendo, portanto, maior que no ultrassom intracoronário: aproximadamente $15 \mu \mathrm{m}$. Contudo, a profundidade da imagem é de aproximadamente 1 a 1,5mm dentro da parede coronária, inferior à obtida com o ultrassom intracoronário.

Desde 2008 uma nova geração de sistemas de OCT está 
disponível para uso, chamada de optical frequency domain. Esta tecnologia permitiu uma velocidade de aquisição de imagens mais rápida, com maior penetração tecidual, sem perda de detalhes vitais ou de resolução comparada à geração anterior. As artérias coronárias podem ser analisadas em poucos segundos, permitindo um uso clinico mais generalizado em um amplo espectro de pacientes e lesões. ${ }^{(35)}$

\subsubsection{Técnica e segurança da tomografia de coerência óptica}

Os cateteres de OCT contém um núcleo gerador de imagem em sua ponta distal que é orientado em um angulo de 90 graus em relação ao comprimento do cateter e que giram ao redor do cateter durante a geração das imagens. Como resultado, as imagens da OCT é exibida como cortes transversais da coronária.

Todos os vasos coronários epicárdicos, assim como os enxertos venosos e arteriais, podem ser analisados pela OCT. ${ }^{(36)}$ A OCT intracoronária é realizada introduzindo-se distalmente um pequeno cateter de imagem (2,7 French) sobre um fio-guia (de 0,014 polegadas) por meio de cateteres-guias tradicionais ( 6 French ou maiores). Uma tração motorizada é realizada para examinar todo o segmento coronário desejado. A tração é tipicamente de 20 milímetros por segundo com uma taxa de quadros igual ou superior a 100 quadros por segundo. Como o sangue dispersa o sinal da OCT, ele é temporariamente removido com a injeção de meio de contraste durante a duração da tração da OCT (3,0 mililitros por segundo).

Como os atuais sistemas de OCT permitem a aquisição das imagens de maneira muito rápida, a possível indução de isquemia pela necessidade de ausência de sangue para a aquisição não costuma ocorrer. Em um estudo envolvendo 144 analises de OCT em 90 pacientes, o procedimento obteve sucesso em 89. Um paciente apresentou espasmo coronário transitório que se resolveu com administração de nitrato intracoronário. Nenhum pacientes apresentou nefropatia induzida por contraste ou complicações maiores. ${ }^{(37)}$ 


\subsubsection{Imagens da tomografia de coerência óptica}

As imagens da OCT mostram a parede da artéria coronária normal como uma estrutura circular com três camadas concêntricas. A camada mais interna rica em sinal reflete a membrana elástica interna, a camada do meio mais escura representa a média e a camada mais externa rica em sinal representa a membrana elástica externa. ${ }^{(38)}$

A OCT tem a habilidade de caracterizar a estrutura e a extensão da doença coronária com grande detalhamento. Os diversos componentes da placa aterosclerótica tem propriedades ópticas diferentes. Normalmente, as imagens da OCT são interpretadas por avaliação visual da intensidade e geometria do sinal. A concordância inter e intraobservador para caracterização visual da placa aterosclerótica mostrou-se adequada. ${ }^{(39,40)}$

Quando lesões ateroscleróticas estão presentes, a OCT pode fornecer detalhes da composição do tecido. Placas ateroscleróticas fibróticas costumam ser ricas em colágeno ou células musculares e ter sinais de OCT homogêneos. Calcificações dentro das placas ateroscleróticas são identificadas pela presença de regiões bem delineadas, com pouca dispersão reversa, fraca em sinal e heterogênea. Núcleos necróticos ou tecidos rico em lipídeos são menos bem delineados que as calcificações, aparecendo como regiões com bordas difusas de baixo sinal, sobrepostas por uma faixa rica em sinal, correspondente à capa fibrosa. A superioridade da OCT para detecção de placa aterosclerótica rica em lipídeos foi confirmada em estudos comparando a OCT, o ultrassom intracoronário e técnicas ultrassonográficas de analise da composição da placa aterosclerótica. ${ }^{(38,41)}$

A OCT permite o diagnostico do fibroateroma de capa fina com uma sensibilidade de $90 \%$ e uma sensibilidade de $79 \%$ quando comparado com a histopatologia, ${ }^{(42)}$ assim como permite uma medida acurada da espessura da capa fibrosa, com baixa variabilidade. ${ }^{(43)}$

Outras estruturas também observadas são acúmulos locais de macrófagos e cristais de colesterol e neovascularização. Macrófagos podem vistos como pontos ricos em sinal, distintos ou confluentes, que excedem a intensidade de ruído do segundo plano. Eles podem ser vistos no limite entre o fundo da capa fibrosa e o começo do núcleo necrótico. ${ }^{(44)}$ Os cristais de colesterol são observados 
como uma região linear fina com alta intensidade de sinal dentro de uma placa lipídica. ${ }^{(45)} A$ neovascularização dentro da camada intima aparece como um vazio pobre em sinal que são nitidamente delineadas e normalmente são vistas em múltiplos quadros contíguos. ${ }^{(46)}$

\subsection{Angiotomografia computadorizada coronária}

O racional para o desenvolvimento de métodos não-invasivos de avaliação da anatomia coronária provém em sua maior parte da necessidade de se melhorar a segurança e a custo-efetividade. A visualização não-invasiva das coronárias é considerada difícil em virtude de seu pequeno calibre, de seu percurso tortuoso e de estar em constante movimento. Assim, as modalidades de imagem não-invasivas devem potencialmente possuir uma alta resolução temporal e espacial.

\subsubsection{Tecnologia da angiotomografia computadorizada coronária}

A angiotomografia computadorizada coronária (angioTC) é um exame bastante disponível e minimamente invasivo, que pode ser realizado em tomografias computadorizadas de 64 ou mais detectores (TCMD).

Tomógrafos de diferentes fabricantes variam pouco em suas especificações técnicas, mas todas têm dois fatores em comum: alta resolução temporal, suficiente para gerar imagens do coração em batimento sem artefatos de movimento, quando a frequência cardíaca se mantém regular e lenta [geralmente menor que 60 a 70 batimentos por minuto (bpm)]; e aquisição de mais de um corte transversal da imagem por rotação dos detectores.

A resolução temporal da TCMD é definida pelo tempo de rotação de seus detectores e reflete a capacidade de congelar o movimento cardíaco, produzindo imagens nítidas. As TCMD usadas para angioTC devem ter o menor tempo de rotação do tubo de raio $X$ possível, sendo o dos equipamentos de ultima geração, com 320 detectores, igual ou inferior a 350 milisegundos (mseg), correspondente a uma resolução temporal ao redor da metade deste valor. 
A tecnologia da TCMD tem evoluído rápida e continuamente. A maioria das TCMD hoje em uso tem ao menos 64 detectores, uma colimação de $0,6 \mathrm{~mm}$ e uma rotação de $400 \mathrm{mseg}$ (correspondente a uma resolução temporal de aproximadamente $200 \mathrm{mseg}$ ). ${ }^{(47,48)}$ A mais nova geração de TCMD pode adquirir 320 cortes transversais a cada rotação.

A angioTC realizada na tomografia computadorizada de 320 detectores (TC320) permite imagens volumétricas tridimensionais de todo o coração durante a diástole de um único ciclo cardíaco. Assim, o coração inteiro pode ser visualizado com uniformidade temporal (isto é, ao mesmo tempo, sem atraso temporal da base ao ápice). A TC320 tem uma resolução temporal padrão de aproximadamente $175 \mathrm{mseg}$, metade do tempo de rotação do detector, porém mais longo do que os $33 \mathrm{mseg}$ da cinecoronariografia, que opera a 30 quadros por segundo. ${ }^{(49)}$ Este equipamento possibilita a diferenciação dos subtipos de placas ateroscleróticas (calcificadas, não calcificadas ou mistas) e a quantificação da porcentagem de estenose, além de requerer menor tempo para aquisição das imagens e menores doses de radiação quando comparado às gerações de TCMD prévias. ${ }^{(50-52)}$

\subsubsection{Técnica e segurança da angiotomografia computadorizada coronária}

$\mathrm{Na}$ angioTC coronária, o contraste iodado endovenoso é administrado em bolus. Imagens da passagem do contraste pelas coronárias são, então, adquiridas em compasso com o eletrocardiograma. Beta-bloqueadores orais e endovenosos podem ser administrados para diminuir a frequência cardíaca idealmente ao redor de $60 \mathrm{bpm}$. Nitroglicerina sublingual pode ser utilizada imediatamente antes do exame para atingir máxima vasodilatação.

O grau de redução luminal na angioTC foi determinado, na maioria dos estudos, por estimação visual. Estudos realizados utilizando TCMD proporcionaram melhor qualidade das imagens e melhor acurácia diagnóstica à medida que se usavam máquinas de geração mais atuais (devido ao aumento no numero de detectores, à diminuição na espessura dos cortes transversais e à maior velocidade da aquisição das imagens). ${ }^{(53)} \mathrm{A}$ angioTC é capaz de detectar estenoses coronárias clinicamente significantes (maior ou igual a 50\%) com alta acurácia 
(sensibilidade de $85 \%$ e especificidade de $90 \%$ ). ${ }^{(54)}$

Os valores preditivos positivos e negativos da angioTC para detectar estenoses significativas quando comparada à cinecoronariografia variam com a prevalência da doença na população estudada. Em estudos multicêntricos, os valores preditivos positivos variaram de 64 a $91 \%$ e os negativos, de 83 a $99 \% .{ }^{(54-56)}$

Alguns fatores relacionados ao paciente podem interferir na qualidade diagnóstica das imagens tomográficas, tais como: frequência cardíaca alta, ritmo cardíaco irregular (fibrilação atrial), incapacidade de interrupção da respiração por alguns segundos, presença de calcificação coronária importante e presença de stents.

A injeção de contraste iodado é necessária para a angioTC. $\mathrm{Na}$ maioria dos casos, 60 a $120 \mathrm{~mL}$ de contraste são injetados a uma velocidade de 4 a $5 \mathrm{~mL} / \mathrm{min}$. Dessa maneira, a angioTC tem como contraindicação absoluta os pacientes com história de alergia ao contraste iodado e como relativa os pacientes com alto risco de nefropatia por contraste.

Apesar das novas gerações de TCMD serem capazes de realizar o exame com menor dose de radiação para os pacientes, o risco relacionado à radiação ainda existe. ${ }^{(50-52)} \mathrm{O}$ potencial cancerígeno secundário à realização de imagens cardiovasculares é controverso e ainda precisa ser comprovado. Como todo estudo utilizando radiação ionizante, a angioTC deve ser indicada após avaliação individualizada do risco e do benefício de cada paciente.

\subsubsection{Imagens da angiotomografia computadorizada coronária}

A capacidade de detecção de placas ateroscleróticas nãocalcificadas subclínicas moderadamente obstrutivas através da angioTC coronária, assim como as possíveis implicações destas placas no risco cardiovascular, são motivo de diversos estudos. ${ }^{(57)}$

Baseados na perspectiva de que a TCMD poderia auxiliar na prevenção de SCA ao detectar placas ateroscleróticas, e em especial as vulneráveis, estudos clínicos avaliaram a capacidade da TCMD em detectar tais placas, usando o ultrassom intracoronário como comparação padrão. ${ }^{(57-61)}$ Estes estudos mostraram que a placa aterosclerótica vulnerável é associada a remodelamento positivo, baixa 
atenuação na TCMD e pequenas calcificações localizadas. Quando identificadas em pacientes com fatores de risco ateroscleróticos, estas características permitiram predizer a ocorrência de uma SCA subsequente (razão de risco: 22,8). ${ }^{(61)}$ Observouse também, a associação da placa aterosclerótica vulnerável com um padrão especifico de atenuação da placa aterosclerótica chamado sinal do anel de guardanapo ("napkin-ring sign"). Trata-se de uma placa aterosclerótica com baixa atenuação cercada por uma área marginal de maior atenuação (Figura 1). Contudo, apesar do ultrassom intracoronário ter se mostrado um método muito sensível para detectar o conteúdo e o volume de cálcio na placa aterosclerótica, bem como para definir o tamanho do vaso, este não se mostrou preciso para avaliar o conteúdo lipídico das placas ateroscleróticas nem de estruturas de menor resolução associadas à placa aterosclerótica vulnerável, como o fibroateroma de capa fina. ${ }^{(7,60)}$

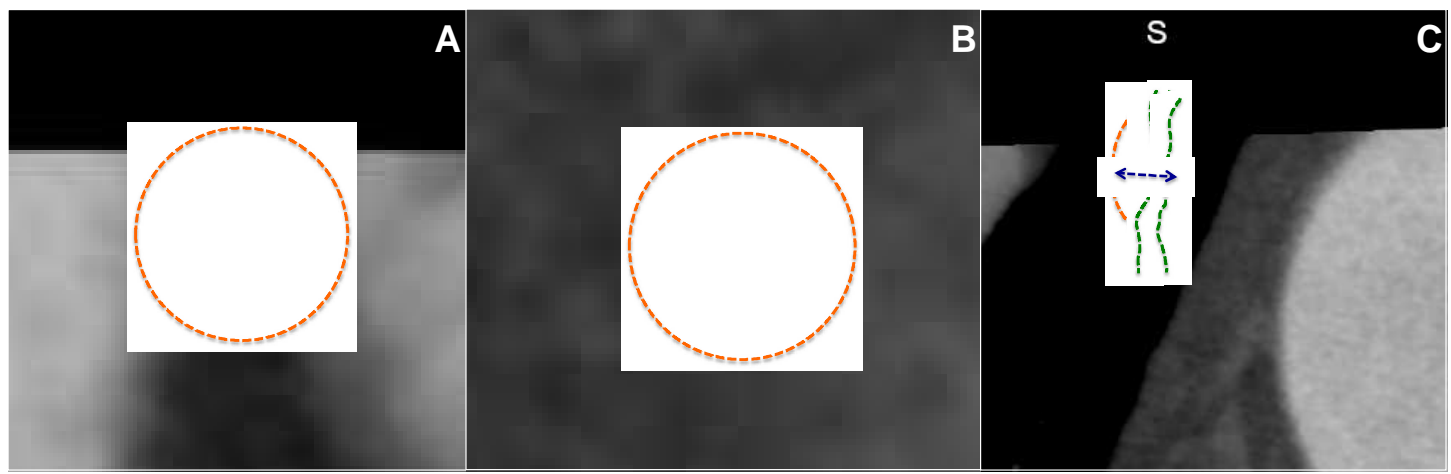

A) calcificação localizada (em azul), definida com um ponto de cálcio dentro da placa; sinal do anel de guardanapo, definido como uma placa de baixa atenuação central (em amarelo) circundada por tecido de alta atenuação (em vermelho); B) placa de baixa atenuação (em amarelo), definida como uma placa não calcificada com atenuação interna inferior a $30 \mathrm{HU}$; C) remodelamento positivo (em azul), definido como uma razão entre o diâmetro externo do vaso no local da placa dividido pela média do diâmetro externo do vaso proximal e distal maior do que 1,1; A, B e C em verde: luz do vaso; em laranja: parede externa da artéria coronária.

Figura 1. Representação das características tomográficas de vulnerabilidade

\subsection{Motivação do estudo}

Centenas de milhares de pessoas morrem ou sofrem um IAM anualmente sem que tenham sido previamente diagnosticadas com doença arterial coronária. Por este motivo, o objetivo de futuras investigações deveria ser a identificação de indivíduos assintomáticos ou pouco sintomáticos com a presença de placas ateroscleróticas coronarianas que sejam potencialmente vulneráveis. Existe a necessidade de se validar uma modalidade não-invasiva de imagem para 
identificação destes tipos de placa aterosclerótica, a fim de que as vantagens do tratamento médico preventivo intensivo possam ser inteiramente obtidas.

A variabilidade existente na acurácia das imagens obtidas pela TCMD para caracterização da placa aterosclerótica pode impedir seu uso generalizado para este fim. Até o presente momento, não foram publicados estudos comparativos entre as imagens obtidas pela TC320 e pela OCT. Este estudo visa avaliar a capacidade de caracterização das placas ateroscleróticas coronárias pelas imagens obtidas pela angioTC coronária de 320 detectores TC320 e compará-la às imagens obtidas pela OCT. Isso tornaria possível o avanço do conhecimento e o consequente tratamento, das doenças arterioscleróticas.

Soeda et al. ${ }^{(62)}$ compararam as imagens de OCT com a acurácia diagnóstica da tomografia computadorizada de dupla fonte de raio $X$ (TCDFRX) na caracterização tecidual de placas ateroscleróticas não-estenóticas e não-culpadas em pacientes com SCA. Também avaliaram se a TCDFRX é capaz de identificar o fibroateroma de capa fina diagnosticado pela OCT. Os autores avaliaram 17 pacientes, nos quais um total de 162 regiões coronárias foram estudadas. Os valores médios da placa aterosclerótica fibrosa, com núcleo lipídico e com deposito de cálcio pela TCDFRX foram, respectivamente, 7,5, 28,9, e 515,9 Houndsfield Units $(\mathrm{HU})$. Placas ateroscleróticas com depósito de cálcio localizado foram detectadas com sensibilidade de $88,9 \%$ e especificidade de $98.6 \%$, enquanto que a placa aterosclerótica com núcleo lipídico foi detectada com menor sensibilidade, 73,1\%, e maior especificidade, 94\%. Nos fibroateromas de capa fina, as medidas das áreas transversais da placa aterosclerótica foram significativamente maiores em comparação com medidas de placas ateroscleróticas ricas em lipídeo com capa fibrosa espessa. Por outro lado, o valor médio de atenuação da placa aterosclerótica foi significativamente menor, mas a calcificação concomitante foi mais frequentemente observada.

Os mesmos autores afirmam que a TCDFRX é útil na avaliação não-invasiva de tecidos calcificados e fibrolipídicos na placa aterosclerótica coronária e que a combinação dos parâmetros acima citados parece fornecer um útil índice não-invasivo para a diferenciação dos fibroateromas de capa fina daqueles fibroateromas que têm capa espessa, muito embora a TCDFRX não tenha sido capaz de identificar especificamente o fibroateroma de capa fina. ${ }^{(62)}$

Kashiwagi et al. ${ }^{(63)}$ investigaram a capacidade da angioTC 
coronária de 64 detectores em detectar os fibroateromas de capa fina, já identificados pela OCT. Eles dividiram sua população de estudo em 2 grupos, de acordo com os achados da OCT [grupo com fibroateroma de capa fina $(n=25)$ e grupo sem fibroateroma de capa fina $(n=80)]$ e estudaram apenas as lesões consideradas culpadas. A SCA estava presente em 31 dos 105 pacientes. O remodelamento positivo e o sinal do anel de guardanapo (identificados pela TCMD de 64 detectores) foram mais frequentemente detectados no primeiro grupo. Os valores de PCR também foram maiores no primeiro grupo. Já os valores de atenuação da TCMD foram menores no grupo com fibroateroma de capa fina. A sensibilidade, a especificidade, os valores preditivos positivo e negativo do sinal do anel de guardanapo em detectar fibroateroma de capa fina foram 44\%, 96\%, 79\% e $85 \%$, respectivamente. Os autores sugeriram que este sinal passasse a ser considerado um marcador sensível na detecção de fibroateroma de capa fina, tendo em vista o atual aumento da resolução das TCMD.

Outro estudo comparou sistematicamente as imagens obtidas pela TCMD (64 detectores), pelo ultrassom intracoronário e pela OCT com análises histopatológicas de corações humanos de cadáveres para diferenciar lesões precoces de lesões avançadas. ${ }^{(64)}$ Os achados de composição da placa aterosclerótica e grau de lesão, da TCMD e do ultrassom intracoronário, correlacionaram-se moderadamente com os achados histopatológicos, enquanto que os achados da OCT se correlacionaram fortemente à histopatologia. A OCT se mostrou melhor em diferenciar lesões precoces de lesões avançadas quando comparada ao ultrassom intracoronário e à TCMD (área sob a curva: 0.858 [95\% IC: 0,802, 0,913], 0,631 [95\% IC: 0,554, 0,709], e 0,679 [95\% IC: 0,618, 0,740]), respectivamente. Além disso, observou-se que a concordância interobservador foi excelente para a OCT e a TCMD, sendo apenas boa para o ultrassom intracoronário.

Wieringa et al. $^{(65)}$ investigaram a capacidade da angioTC coronária de 64 detectores e do ultrassom coronário em diferenciar as características da placa aterosclerótica quando comparados à OCT. Para isto, estudaram as imagens de 30 pacientes com IAM sem supradesnivelamento do segmento ST em dois níveis: da lesão culpada como um todo $(n=30)$ e de cortes transversais da lesão culpada $(n=1083)$. Eles observaram que achados normais tanto na angioTC quanto no ultrassom coronário foram associados a placa aterosclerótica precoce (isto é, sem lipídeo pela OCT). No ultrassom, placas 
ateroscleróticas calcificadas e lipídicas foram associadas com placa aterosclerótica avançada (com lipídeo pela OCT).

Tomizawa et al. ${ }^{(66)}$ compararam a acurácia de diferentes modelos para predizer o diagnóstico de fibroateroma de capa fina usando parâmetros da angioTC coronária de 64 detectores, validados pela OCT: modelo 1 incluía índice de remodelamento maior que 1,1 , atenuação menor que $30 \mathrm{HU}$ e sinal do anel de guardanapo; modelo 2, índice de remodelamento maior que 1,1, atenuação menor que $30 \mathrm{HU}$ ou sinal do anel de guardanapo; e modelo 3, que utilizou um modelo de regressão usando o índice de remodelamento, a proporção do volume de baixa atenuação e o sinal do anel de guardanapo. Após analisar 129 placas ateroscleróticas em 106 pacientes, foram encontradas 83 placas ateroscleróticas com fibroateroma de capa fina. O modelo 3 mostrou uma área sob a curva de 0,96, maior que os demais modelos $(1: 0,74 ; 2: 0,72 ; p<0,01)$. A acurácia do modelo 3 (93\%) também foi maior que os demais (1: $67 \% ; 2: 80 \% ; p<0,01)$, sendo sua sensibilidade e especificidade $94 \%$ e $91 \%$, respectivamente.

Mais recentemente, Yang et al. ${ }^{(67)}$ avaliaram se as características da placa aterosclerótica avaliadas pela angioTC coronária eram associadas com à presença de fibroateroma de capa fina definido pela OCT. Foram analisados 727 cortes transversais em 31 lesões de 28 pacientes. Cortes transversais com fibroateroma de capa fina pela OCT apresentaram uma frequência maior de placa aterosclerótica de baixa atenuação, sinal do anel de guardanapo, remodelamento positivo e alta carga de placa aterosclerótica na angioTC coronária quando comparados aos cortes transversais sem fibroateroma de capa fina. Após análise multivariável, placa aterosclerótica de baixa atenuação e sinal do anel de guardanapo continuaram associados ao fibroateroma de capa fina (odds ratio de 4,$05 ; p<0,001$, e de 2,47; $p=0,005$, respectivamente). A área do lúmen nas imagens obtidas pela angioTC coronária se correlacionou bem com aquela observada nas imagens obtidas pela OCT $(R=0,859)$. 


\subsection{Objetivos}

1. Caracterizar a presença e os componentes da placa aterosclerótica coronária analisada através das imagens obtidas pela tomografia computadorizada de 320 detectores (método de imagem não-invasivo) e comparálas às imagens obtidas através da tomografia de coerência óptica (método de imagem invasivo intravascular, padrão-ouro).

2. Avaliar se a tomografia computadorizada de 320 detectores é capaz de diferenciar de maneira não-invasiva os fibroateromas dos não-fibroateromas de forma invasiva pela tomografia de coerência óptica. 


\section{MÉTODOS}

\subsection{Aspectos éticos}

Projeto aprovado pelo Sistema Gerenciador de Projetos de Pesquisa em (SGPP №2316-15 30/04/2015) e pelo Comitê de Ética em Pesquisa em (CEP № 45116615.8.0000.0071 28/07/2015) da instituição.

\subsection{Seleção da amostra}

Conduzimos um estudo transversal unicêntrico retrospectivo no qual foram selecionados todos os pacientes do banco de dados do Serviço de Cardiologia Intervencionista do Hospital Israelita Albert Einstein que haviam realizado TC320 e OCT na instituição de novembro de 2012, quando foi incorporada a OCT na instituição, a dezembro de 2015. O intervalo máximo aceito entre a realização da TC320 e da OCT foi de 30 dias. O tamanho amostral necessário foi calculado em 56 segmentos coronários, estimando-se a necessidade de inclusão de um total de 28 sujeitos de pesquisa.

Os seguintes critérios de exclusão foram adotados: (i) segmentos com intervenção prévia (percutâneo ou cirúrgico), (ii) imagens inadequadas adquiridas pela TC320 ou pela OCT (ausência ou perda de definição nas imagens de 360 graus e/ou características teciduais caracterizadas em menos de $0,66 \mathrm{~mm}$ de comprimento longitudinal) e (iii) realização da TC320 e da OCT com intervalo maior do que 30 dias.

\subsection{Coleta de dados}

Foram coletadas as informações sobre a idade (anos), gênero (masculino, feminino), índice de massa corpórea (IMC, $\mathrm{kg} / \mathrm{m}^{2}$ ), quadro clínico, tabagismo, hipertensão, diabetes, dislipidemia, insuficiência renal, IAM prévio, revascularização miocárdica previa e dosagem de proteína C reativa préprocedimento. 


\subsection{Estudos de imagem}

As imagens da OCT foram obtidas conforme o protocolo descrito a seguir: após a realização da cinecoronariografia, administrou-se medicação anticoagulante (heparina 70-100ui/kg) e vasodilatadora intracoronária (nitroglicerina

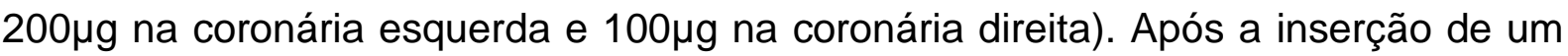
fio-guia na vaso-alvo, um cateter intravascular de OCT tipo Frequency-Domain (C7 $\mathrm{XR}^{\mathrm{TM}}$ Intravascular Imaging Catheter, St Jude Medical, Saint Paul, Minnesota, USA) é inserido até o segmento distal através de um cateter maior ou igual a 6 French. As imagens são adquiridas utilizando o método de fluxo continuo e uma tração automática de $1,0 \mathrm{~mm} / \mathrm{seg}$.

As imagens da TC320 foram realizadas utilizando-se 0 aparelho Aquilion One, Toshiba Medical, Tochigi-ken, Japão, que apresenta 320 fileiras de detectores de $0,5 \mathrm{~mm}$ e tempo de rotação do tubo de $350 \mathrm{mseg}$. As imagens foram obtidas segundo o protocolo descrito a seguir: pacientes cuja frequência cardíaca era superior a $60 \mathrm{bpm}$, exceto em caso de contraindicações, receberam, antes do exame ser realizado, medicação betabloqueadora por via endovenosa e/ou por via oral. Nitrato sublingual também foi administrado em todos os pacientes na ausência de contraindicações. Inicialmente, para avaliação das placas ateromatosas coronarianas calcificadas, foram realizadas imagens com $3 \mathrm{~mm}$ de espessura sem uso de contraste endovenoso. Posteriormente, para avaliação luminal das artérias coronárias, foi realizada a angioTC com contraste endovenoso, injetado através de um acesso periférico. As imagens completas do coração foram obtidas em 1 ou 2 batimentos cardíacos.

Durante a aquisição das imagens foi realizado o registro do eletrocardiograma para sincronização prospectiva da reconstrução dos dados, habitualmente reconstruídos entre 70 e $80 \%$ do intervalo $R$ - $R$ para os pacientes com frequência cardíaca de até $65 \mathrm{bpm}$ e entre 30 e $80 \%$ do intervalo R-R para os pacientes com frequência cardíaca maior de $65 \mathrm{bpm}$. A voltagem do tubo e a corrente foram ajustadas de acordo com o IMC de cada paciente.

Foram injetados $60-80 \mathrm{~mL}$ de contraste iodado não iônico, com concentração de $350 \mathrm{mg} / \mathrm{mL}$ em uma veia antecubital, com uma velocidade de 5,0 a $6,0 \mathrm{~mL} / \mathrm{seg}$, seguidos de $30 \mathrm{~mL}$ de soro fisiológico $0,9 \%$ na mesma velocidade. Para 
ajustar o início da aquisição das imagens com a chegada do bolus de contraste, foram adquiridas imagens estacionárias capazes de avaliar automaticamente o aumento da densidade no interior da aorta descendente. As imagens foram adquiridas durante uma apnéia inspiratória de até 5 segundos. Foram realizadas reconstruções das imagens em intervalos de 5 a $10 \%$ do $R-R$ ou no momento de menor quantidade de movimentos.

\subsection{Método de leitura}

\subsubsection{Coregistro das imagens}

Para se estabelecer que as mesmas regiões coronárias estavam sendo analisadas na TCO e na TC320, uma imagem longitudinal específica da TCO era reconstruída, tendo por base marcos anatômicos observados na cinecoronariografia (óstio do vaso e/ou seus subramos), e segmentada, tendo por base os segmentos coronarianas propostos pelo estudo CORE-64. ${ }^{(68)}$ Caso não houvesse um marco distal ou proximal claro, definia-se uma distancia pelo tamanho da corrida da TCO a partir do marco anatômico contrário. O segmento obtido na TCO era delimitado em uma imagem da cinecoronariografia, que era utilizada para definir o segmento a ser estudado na TC320 (Figura 2). 


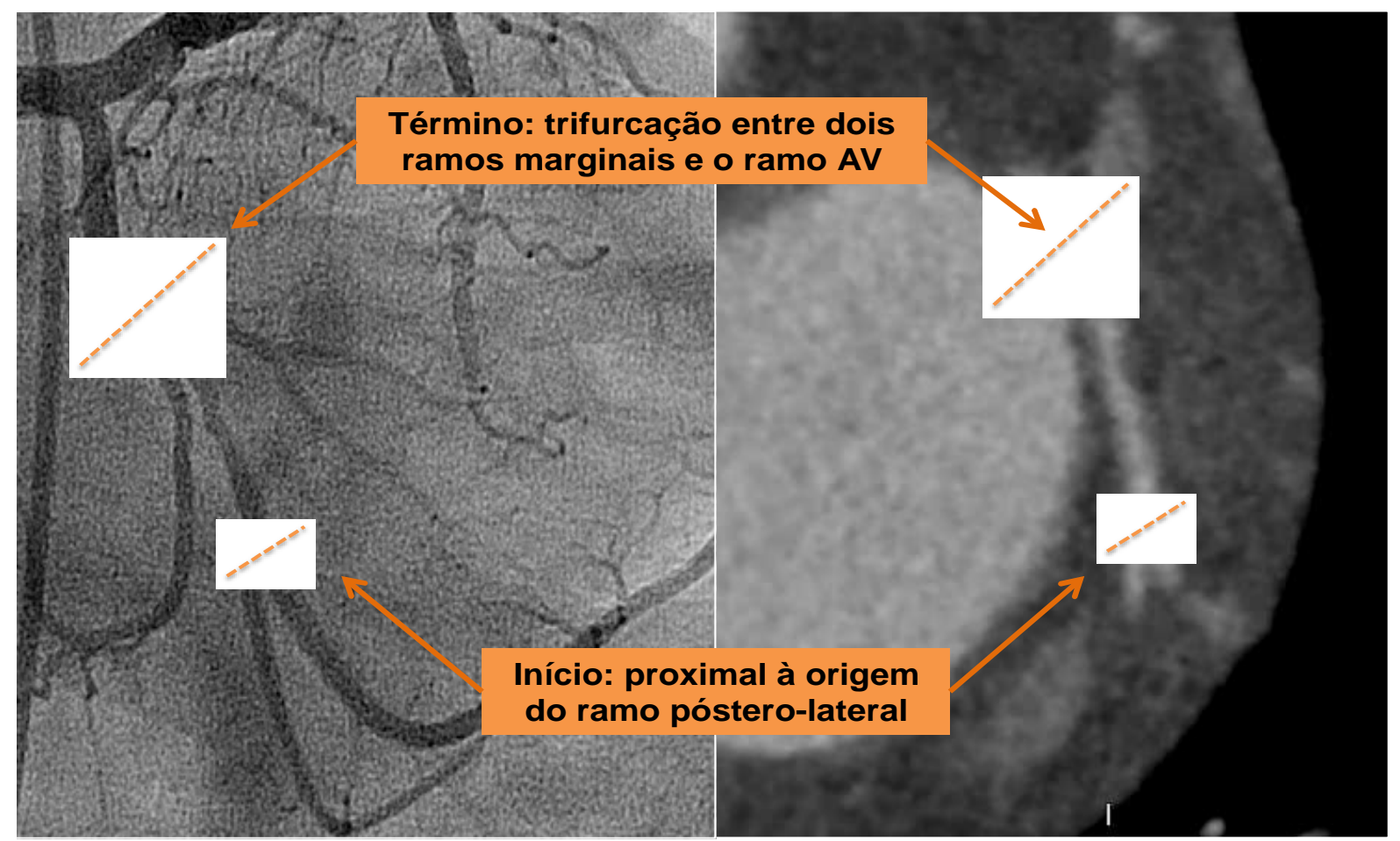

Figura 2. Modelo de coregistro: o segmento obtido na tomografia de coerência óptica era delimitado na cinecoronariografia e esta era utilizada para definir o segmento na tomografia computadorizada de 320 canais

\subsubsection{Leitura das imagens}

A análise das imagens obtidas pela OCT foi realizada com o programa QIVUS® versão 3.0 (Medis Medical Imaging, Leiden, Netherland) em um Corelab (Cardiovascular Research Center, São Paulo, SP, Brasil) independente e "cego", ou seja, que desconhecia tanto as imagens da TC320 quanto os parâmetros clínicos dos pacientes.

A análise das imagens obtidas pela TC320 foi realizada com o programa Vitrea FX $1.0 \AA$ (Vital Images, Minnetonka, Mn, EUA) e em consenso por dois radiologistas especialistas experientes e "cegos", ou seja, que desconheciam tanto as imagens da OCT e quanto os parâmetros clínicos dos pacientes.

\subsubsection{Parâmetros quantitativos e qualitativos}

As análises da OCT foram realizadas de acordo com definições do Consenso de 2012 do Grupo de Trabalho Internacional para a padronização e 
validação da OCT. ${ }^{(45,69)}$ As seguintes medidas foram aferidas nas imagens obtidas da OCT:
I. Área luminal mínima
II. Diâmetro luminal mínimo
III. Índice de excentricidade [(diâmetro luminal mínimo - diâmetro luminal máximo) / diâmetro luminal máximo]

I. Assimetria do lúmen (menor diâmetro mínimo / maior diâmetro máximo)

II. Percentual de estenose do lúmen: primeiramente obtêm-se a média das referências através da média entre a área do lúmen do primeiro e do último frame analisado; a seguir, calcula-se o percentual de estenose do lúmen: (média das referências - área mínima do lúmen) / média das referências x 100)

\section{Espessura da capa fibrosa}

IV. Angulação do conteúdo lipídico nos fibroateromas

Os segmentos coronários obtidos pela OCT foram classificados hierarquicamente de acordo com o pior fenótipo da placa aterosclerótica: coronária normal < placa aterosclerótica fibrocalcificada < placa aterosclerótica fibrótica < fibroateroma. Também foi analisada a presença de macrófagos, de neovascularização e/ou de cristais de colesterol.

As análises das imagens obtidas pela angioTC coronária foram realizadas de acordo com a recente classificação proposta pelo Consenso da Sociedade de Tomografia Computadorizada Cardiovascular, ${ }^{(70)}$ chamada Coronary Artery Disease Reporting and Data System (CAD-RADS). Em suma, este modelo leva em consideração o grau máximo de estenose coronária dentro do segmento analisado, o local das estenoses, e a presença de características tomográficas de vulnerabilidade da placa aterosclerótica (remodelamento positivo, placa aterosclerótica de baixa atenuação menor que $30 \mathrm{HU}$, calcificação localizada e sinal do anel de guardanapo). A placa aterosclerótica é considerada vulnerável caso apresente duas ou mais características tomográficas de vulnerabilidade.

As seguintes medidas foram realizadas nas imagens obtidas pela TC320:
I. Área luminal mínima
II. Diâmetro luminal médio
III. Diâmetro luminal mínimo 
IV. Diâmetro luminal máximo

V. Grau de estenose

O grau de estenose foi calculado e categorizado de acordo com o proposto pelo CAD_RADS: ${ }^{(70)}$ CAD-RADS 0 (0\%): sem estenose visível; CAD-RADS 1 (1-24\%): estenose mínima; CAD-RADS 2 (25-49\%): estenose leve; CAD-RADS 3 (50-69\%): estenose moderada; e CAD-RADS 4 (70-99\%): estenose grave.

As placas ateroscleróticas foram analisadas de acordo com sua composição na angioTC coronária: placas ateroscleróticas calcificadas (placas apenas com estruturas de alta densidade quando comparadas com o contraste intraluminal); placas ateroscleróticas não-calcificadas (placas com menor densidade quando comparadas com o contraste intra-luminal); e placas ateroscleróticas mistas (presença de componentes calcificados e não-calcificados numa única placa). Os segmentos coronários obtidos pela angioTC coronária foram classificados hierarquicamente de acordo com o pior fenótipo da placa aterosclerótica: coronária normal < placa aterosclerótica calcificada < placa aterosclerótica mista < placa aterosclerótica não-calcificada. Também foram analisadas os seguintes parâmetros tomográficos de vulnerabilidade da placa aterosclerótica: placa aterosclerótica de baixa atenuação $(<30 \mathrm{HU})$, remodelamento positivo, calcificação localizada e sinal do anel do guardanapo.

A comparação entre as imagens das placas ateroscleróticas obtidas pelos métodos de imagem descritos foi realizada primeiramente considerando-se a classificação de placas ateroscleróticas calcificadas e não calcificadas pela TC320 e, num segundo momento, considerando a classificação de fibroateroma e não fibroateroma pela OCT.

\subsection{Análise estatística}

\subsubsection{Cálculo amostral}

Para o cálculo do tamanho da amostra foram considerados, inicialmente, os achados descritos por Soeda et al. ${ }^{(62)} \mathrm{O}$ cálculo amostral para a concordância entre dois avaliadores utilizando o coeficiente Kappa de Cohen foi realizado buscando utilizar intervalos de confiança com amplitudes de 0,30; a 
metodologia proposta em Altman (1990) foi utilizada. Com base nesses parâmetros, o tamanho amostral foi estimado em 56 segmentos analisados. Para isto, estimamos a inclusão de 28 pacientes (pelo menos 2 segmentos coronários analisados por paciente) neste estudo.

\subsubsection{Análise dos dados}

As medidas lineares foram expressas em milímetros $(\mathrm{mm})$, as medidas de área em milímetros quadrados $\left(\mathrm{mm}^{2}\right)$, e as medidas angulares em graus $\left({ }^{\circ}\right)$. Os resultados das variáveis qualitativas foram descritos por frequências relativas e absolutas. Os resultados das variáveis quantitativas foram descritos por médias e desvios-padrão (DP) ou medianas e intervalos interquartis, onde apropriado. Associações entre duas variáveis foram verificadas por meio de testes exatos de Fisher, por testes de Mann-Whitney e por teste $t$ de Student, onde apropriado. As distribuições das medidas numéricas foram verificadas por meio de histogramas e boxplots.

Para medir a concordância entre os dois métodos foram utilizados os coeficientes de correlação intraclasse ICC3 (two-way mixed model). Ao considerar as classes de área e de estenose, mediu-se a concordância nas classificações por meio de coeficientes de concordância AC2 com pesos ordinais, acompanhados de intervalos de confiança de 95\%. Para as análises de associação e concordância, os segmentos foram considerados como medidas independentes.

Para estimar as medidas de acurácia foram utilizadas equações de estimação generalizadas. A informação dos indivíduos foi incluída no ajuste para se considerar a possível dependência entre suas diversas medidas. Utilizou-se uma estrutura de correlação do tipo permutável, em função dos dados serem organizados em conglomerados. As medidas diagnósticas foram obtidas através de probabilidades estimadas pelos modelos de equações de estimação generalizadas.

As análises foram conduzidas no programa computacional $R(R$ Core Team, 2017), versão 3.4.1 e IBM SPSS (IBM Corp., 2016). Adotou-se nível de significância de $5 \%$. 
3 RESULTADOS

\subsection{Características da amostra}

Encontramos no banco de dados de intervenção coronária do Setor de Cardiologia Intervencionista da Instituição 347 pacientes que haviam realizado angioTC coronária. Identificamos também 120 pacientes que haviam realizado realizado OCT. Desta população, 90 pacientes realizaram ambos os exames, angioTC coronária e OCT. Destes, 52 foram excluídos pelos seguintes motivos: realização de angioTC coronária fora da instituição $(n=16)$, realização de angioTC coronária diferente de 320 detectores $(n=4)$, OCT não ter sido capaz de cruzar a lesão estenótica $(n=4)$, OCT ter sido realizada em segmento intra-stent $(n=14)$, e o tempo entre a angioTC de 320 detectores e a OCT ter sido maior que 30 dias $(n=14)$. Com isso, foram incluídos 38 pacientes, totalizando 51 segmentos coronários analisáveis. Dentre esses segmentos, 9 foram excluídos devido à má qualidade das imagens [flush inadequado $(n=5)$; co-registro não-possível $(n=2)$; morfologia vascular alterada por pré-dilatação da coronária com balão $(n=1)$; segmento com stent bioabsorvível $(n=1)]$. A amostra final do estudo compreendeu, portanto, um total de 32 pacientes e de 42 segmentos analisados (Figura 3 ). 


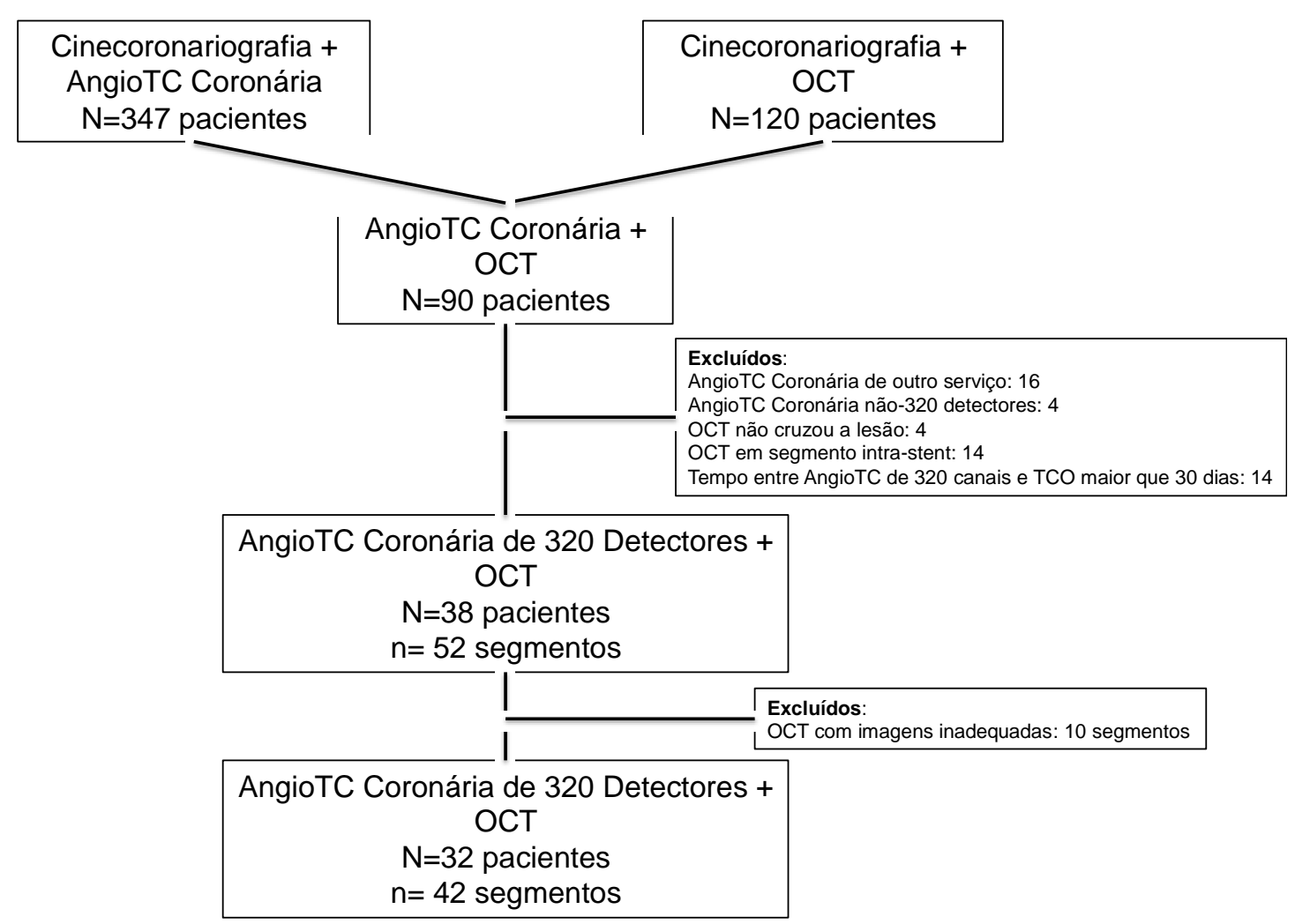

AngioTC coronária de 320 detectores: angiotomografia computadorizada coronária de 320 detectores; OCT: tomografia de coerência óptica.

Figura 3. Fluxograma de distribuição da amostra

Dentre os 32 pacientes incluídos no estudo, a idade média foi de $58 \pm 12$ anos e a maioria era do gênero masculino (84,4\%). Aproximadamente metade dos pacientes apresentavam quadro clinico estável $(56,3 \%)$ e antecedente familiar para doença coronária (56,3\%). Hipertensão arterial estava presente em $50 \%$ de pacientes, diabetes em 18,8\% e dislipidemia em 78,1\%. A mediana do valor de PCR antes da realização da OCT foi 2,2mg/L (Tabela 1). 
Tabela 1. Perfil clínico e demográfico dos pacientes da amostra

\begin{tabular}{lc}
\hline & $\mathbf{N}=\mathbf{3 2}$ \\
\hline Sexo masculino & $27(84,4 \%)$ \\
Idade (anos) & $58 \pm 12$ \\
Peso (Kg) & $88,0(70,5-103,0)$ \\
Altura (cm) & $175 \pm 10$ \\
Índice de Massa Corpórea $\left(\mathrm{Kg} / \mathrm{m}^{2}\right)$ & $27 \pm 4$ \\
Quadro clínico $\mathrm{n}(\%)$ & \\
$\quad$ Assintomático & $14(43,8$ \\
$\quad$ Angina estável & $4(12,5)$ \\
$\quad$ Angina instável & $12(37,5)$ \\
$\quad$ Infarto sem supra ST & $2(6,3)$ \\
Antecedente familiar para doença coronária $\mathrm{n}(\%)$ & $18(56,3)$ \\
Tabagismo n(\%) & $5(15,6)$ \\
Hipertensão arterial $\mathrm{n}(\%)$ & $16(50)$ \\
Diabetes Mellitus n(\%) & $6(18,8)$ \\
Dislipidemia n(\%) & $25(78,1)$ \\
Insuficiência renal $\mathrm{n}(\%)$ & $0(0,0)$ \\
Infarto do miocárdio prévio n(\%) & $0(0,0)$ \\
Angioplastia prévia $\mathrm{n}(\%)$ & $6(18,8)$ \\
Cirurgia cardíaca prévia $\mathrm{n}(\%)$ & $1(3,1)$ \\
Proteína C reativa (mg/L) & $2,2(0,7-6,5)$ \\
\hline Valores apresentados em números absolutos e frequência, média e \\
desvio padrão e mediana e intervalo interquartil.
\end{tabular}

\subsection{Parâmetros quantitativos e qualitativos}

Os resultados quantitativos e qualitativos das imagens obtidas pela OCT e pela TC320 são apresentados na tabela 2. O comprimento médio dos segmentos analisados na imagem obtida pela OCT foi de $28,74 \pm 10,41 \mathrm{~mm}$. A espessura média da capa dos fibroateromas foi $122 \pm 25 \mu \mathrm{m}$.

A área luminal mínima foi de $2,78 \pm 1,23 \mathrm{~mm}$ pela OCT e de $3,29 \pm 1,49 \mathrm{~mm}$ pela TC320 $(p<0,001)$. O diâmetro luminal máximo foi $2,96 \pm 0,53 \mathrm{e}$ $2,24 \pm 0,69$, respectivamente $(p=0,02)$. Os diâmetros luminais mínimo e médio foram semelhantes entre os dois métodos.

Macrófagos foram observados em $73,8 \%$ e cristais de colesterol em $33,3 \%$ dos segmentos das imagens obtidas pela OCT. Cerca de dois terços das placas ateroscleróticas foram caracterizadas como vulneráveis, de acordo com a definição CAD-RADS, nas imagens obtidas pela TC320.

Cerca de metade dos segmentos $(54,8 \%)$ apresentavam placa aterosclerótica moderadamente obstrutiva (entre $50-69 \%$ de estenose) nas imagens obtidas pela OCT, enquanto apenas $38,1 \%$ nas imagens obtidas pela TC320. 
Tabela 2. Variáveis quantitativas e qualitativas avaliadas pela tomografia de coerência óptica e pela tomografia computadorizada de 320 detectores

\begin{tabular}{|c|c|c|c|}
\hline & \multicolumn{2}{|c|}{$\mathrm{N}=42$} & \multirow{2}{*}{ Valor-p } \\
\hline & OCT & TC320 & \\
\hline Comprimento dos segmentos $(\mathrm{mm})$ & $28,74 \pm 10,41$ & & \\
\hline Área luminal média $\left(\mathrm{mm}^{2}\right)$ & $6,14 \pm 2,14$ & & \\
\hline Área luminal mínima $\left(\mathrm{mm}^{2}\right)$ & $2,78 \pm 1,23$ & $3,29 \pm 1,49$ & $<0,001$ \\
\hline Diâmetro luminal médio (mm) & $2,71 \pm 0,47$ & $2,04 \pm 0,56$ & 0,30 \\
\hline Diâmetro luminal mínimo (mm) & $2,46 \pm 0,43$ & $1,85 \pm 0,55$ & 0,22 \\
\hline Diâmetro luminal máximo (mm) & $2,96 \pm 0,53$ & $2,24 \pm 0,69$ & 0,02 \\
\hline Índice de excentricidade do lúmen & $0,17 \pm 0,05$ & & \\
\hline Assimetria do lúmen & $0,40 \pm 0,10$ & & \\
\hline Macrófagos & $31(73,8 \%)$ & & \\
\hline Neovascularização & $6(14,3 \%)$ & & \\
\hline Cristais de colesterol & $14(33,3 \%)$ & & \\
\hline Placa vulnerável (definição CAD-RADS) & & $27(64,3 \%)$ & \\
\hline Sinal do anel de guardanapo & & $22(52,4 \%)$ & \\
\hline Remodelamento positivo & & $28(66,7 \%)$ & \\
\hline Placa de baixa atenuação $(<30 \mathrm{HU})$ & & $16(38,1 \%)$ & \\
\hline Calcificação localizada & & $16(38,1 \%)$ & \\
\hline
\end{tabular}

Valores apresentados em números absolutos e frequência e média e desvio padrão. OCT: tomografia de coerência óptica; TC320: tomografia computadorizada de 320 detectores; CAD-RADS: Coronary Artery Disease Reporting and Data System; HU: Houndsfield units.

\subsection{Comparação entre os métodos}

\subsubsection{Placa aterosclerótica}

A tabela 3 apresenta a descrição da imagem de pior fenótipo de placa aterosclerótica observada concomitantemente em cada um dos métodos. Um segmento de coronária normal observado nas imagens obtidas pela OCT foi considerado como placa aterosclerótica não calcificada nas imagens obtidas pela TC320. A placa aterosclerótica fibrótica foi a mais prevalente nas imagens obtidas pela OCT $(45,2 \%)$, enquanto a placa aterosclerótica mista foi mais frequente nas imagens obtidas pela TC320 (45,2\%). Não foram identificados fibroateromas de capa fina pela OCT em nossa amostra. 
Tabela 3. Descrição fenotípica da placa aterosclerótica observada nas imagens obtidas pela tomografia de coerência óptica e pela tomografia computadorizada de 320 detectores

\begin{tabular}{|c|c|c|c|c|c|}
\hline \multirow[b]{2}{*}{ TC320 } & \multicolumn{4}{|c|}{ OCT } & \multirow[b]{2}{*}{$\begin{array}{l}\text { Total } \\
\mathrm{n}(\%)\end{array}$} \\
\hline & $\begin{array}{c}\text { Normal } \\
\mathrm{n}(\%)\end{array}$ & $\begin{array}{c}\text { Calcificada } \\
\mathrm{n}(\%)\end{array}$ & $\begin{array}{c}\text { Fibrótica } \\
\mathrm{n}(\%)\end{array}$ & $\begin{array}{c}\text { Fibroateroma } \\
\mathrm{n}(\%)\end{array}$ & \\
\hline Normal & $0(0,0)$ & $0(0,0)$ & $0(0,0)$ & $0(0,0)$ & $0(0,0)$ \\
\hline Calcificada & $0(0,0)$ & $4(9,5)$ & $1(2,4)$ & $1(2,4)$ & $6(14,3)$ \\
\hline Mista & $0(0,0)$ & $7(16,7)$ & $6(14,3)$ & $6(14,3)$ & $19(45,2)$ \\
\hline Não calcificada & $1(2,4)$ & $1(2,4)$ & $12(28,6)$ & $3(7,1)$ & $17(40,5)$ \\
\hline Total & $1(2,4)$ & $12(28,6)$ & $19(45,2)$ & $10(23,8)$ & $42(100,0)$ \\
\hline
\end{tabular}

Valores apresentados em números absolutos e frequência. OCT: tomografia de coerência óptica; TC320: tomografia computadorizada de 320 detectores.

Ao dividir-se as placas ateroscleróticas em calcificadas (incluindo as placas ateroscleróticas calcificadas e mistas) e não calcificadas nas imagens obtidas pela TC320, observamos que $44 \%$ das 25 placas ateroscleróticas calcificadas apresentaram calcificação como pior fenótipo nas imagens obtidas pela OCT; das 17 placas ateroscleróticas não calcificadas classificadas nas imagens obtidas pela TC320, 70,6\% foram consideradas como fibróticas nas imagens obtidas pela OCT, havendo uma associação significativa entre as classificações da OCT e da TC320 $(p=0,005)$ (Tabela 4).

Tabela 4. Descrição fenotípica da classificação das placas ateroscleróticas em relação à classificação das placas ateroscleróticas calcificadas e não calcificadas observadas nas imagens obtidas pela tomografia computadorizada de 320 detectores

\begin{tabular}{|c|c|c|c|c|c|c|}
\hline \multirow[b]{2}{*}{ TC320 } & \multicolumn{4}{|c|}{ OCT } & \multirow[b]{2}{*}{$\begin{array}{l}\text { Total } \\
(\%)\end{array}$} & \multirow[b]{2}{*}{ Valor-p } \\
\hline & $\begin{array}{c}\text { Normal } \\
(\%)\end{array}$ & $\begin{array}{c}\text { Calcificada } \\
(\%)\end{array}$ & $\begin{array}{c}\text { Fibrótica } \\
\text { (\%) }\end{array}$ & $\begin{array}{c}\text { Fibroateroma } \\
\text { (\%) }\end{array}$ & & \\
\hline Calcificada & $0(0,0)$ & $11(44,0)$ & $7(28,0)$ & $7(28,0)$ & $25(100,0)$ & 0,005 \\
\hline Não calcificada & $1(5,9)$ & $1(5,9)$ & $12(70,6)$ & $3(17,6)$ & $17(100,0)$ & \\
\hline Total & $1(2,4)$ & $12(28,6)$ & $19(45,2)$ & $10(23,8)$ & $42(100,0)$ & \\
\hline
\end{tabular}

Valores apresentados em números absolutos e frequência e média e desvio padrão; Legenda: OCT: tomografia de coerência óptica; TC320: tomografia computadorizada de 320 detectores.

Mantendo-se a classificação das placas ateroscleróticas em calcificadas e não calcificadas nas imagens obtidas pela TC320 e considerando-se as placas ateroscleróticas das imagens obtidas pela OCT como fibroateroma e não fibroateroma, não encontramos diferença significativa nas proporções de fibroateroma nas placas ateroscleróticas calcificadas e não calcificadas pela TC320 $(p=0,49)$ (Tabela 5). 
Tabela 5. Descrição de fibroateroma pela tomografia de coerência óptica e placa aterosclerótica não calcificada pela angiotomografia computadorizada de 320 detectores

\begin{tabular}{|c|c|c|c|c|}
\hline \multirow[b]{2}{*}{ TC320 } & \multicolumn{2}{|c|}{ OCT } & \multirow{2}{*}{$\begin{array}{l}\text { Total } \\
\mathbf{n}(\%)\end{array}$} & \multirow[b]{2}{*}{ Valor-p } \\
\hline & $\begin{array}{c}\text { Não fibroateroma } \\
n(\%)\end{array}$ & $\begin{array}{c}\text { Fibroateroma } \\
\mathrm{n}(\%)\end{array}$ & & \\
\hline Calcificada & $18(72,0)$ & $7(28,0)$ & $25(100,0)$ & 0,490 \\
\hline Não calcificada & $14(82,4)$ & $3(17,6)$ & $17(100,0)$ & \\
\hline Total & $32(76,2)$ & $10(23,8)$ & $42(100,0)$ & \\
\hline
\end{tabular}

Valores apresentados em números absolutos e frequência e média e desvio padrão; OCT: tomografia de coerência óptica; TC320: tomografia computadorizada de 320 detectores.

\subsubsection{Medidas quantitativas}

A tabela 6 mostra a distribuição das estenoses coronárias entre ambos os métodos de acordo com o sistema CAD-RADS. Na TC320, 73,8\% das placas ateroscleróticas $(n=31)$ mostraram uma estenose superior a $50 \%$. Na OCT, $85,8 \%(n=36)$ apresentaram uma estenose superior a 50\%. Estenoses maiores que $50 \%$ na OCT foram classificadas com menores que 50\% pela TC320 em $21,4 \%$ dos casos. Estenoses menores que 50\% pela OCT foram classificadas como maiores que $50 \%$ pela TC320 em $9,6 \%$ dos casos.

A classificação das estenoses coronárias de acordo com o sistema CAD-RADS mostrou uma acurácia ruim, de 42,1\% [Intervalo de Confiança (IC) $95 \%$ : 25,3 a 58,9], e uma boa concordância ( $A C=0,64$; IC 95\%: 0,46 a 0,82; $p$ $<0,001)$

Tabela 6. Classificação das estenoses de acordo com a classificação pelo sistema CAD-RADS

\begin{tabular}{|c|c|c|c|c|c|c|c|}
\hline \multirow[b]{2}{*}{ OCT } & \multicolumn{5}{|c|}{ TC320 } & \multirow[b]{2}{*}{$\begin{array}{l}\text { Total } \\
\text { n(\%) }\end{array}$} & \multirow[b]{2}{*}{ Valor-p } \\
\hline & $\begin{array}{c}\text { Sem } \\
\text { estenose } \\
(0 \%)\end{array}$ & $\begin{array}{l}\text { Mínima } \\
(0-24 \%)\end{array}$ & $\begin{array}{c}\text { Leve } \\
(25-49 \%)\end{array}$ & $\begin{array}{c}\text { Moderada } \\
(50-69 \%)\end{array}$ & $\begin{array}{l}\text { Grave } \\
(\geq 70 \%)\end{array}$ & & \\
\hline Normal (0\%) & $0(0,0)$ & $0(0,0)$ & $0(0,0)$ & $0(0,0)$ & $0(0,0)$ & $0(0,0)$ & 0,73 \\
\hline Mínima (0-24\%) & $0(0,0)$ & $0(0,0)$ & $1(2,4)$ & $0(0,0)$ & $1(2,4)$ & $2(4,8)$ & \\
\hline Leve (25-49\%) & $0(0,0)$ & $0(0,0)$ & $1(2,4)$ & $2(4,8)$ & $1(2,4)$ & $4(9,5)$ & \\
\hline Moderada (50-69\%) & $0(0,0)$ & $1(2,4)$ & $6(14,3)$ & $10(23,8)$ & $6(14,3)$ & $23(54,8)$ & \\
\hline Grave ( $\geq 70 \%)$ & $0(0,0)$ & $0(0,0)$ & $2(4,8)$ & $4(9,5)$ & $7(16,7)$ & $13(31,0)$ & \\
\hline Total & $0(0,0)$ & $1(2,4)$ & $10(23,8)$ & $16(38,1)$ & $15(35,7)$ & $42(100,0)$ & \\
\hline
\end{tabular}

Valores apresentados em números absolutos e frequência (\%); OCT: tomografia de coerência óptica; TC320: tomografia computadorizada de 320 detectores. 
A concordância entre as medidas de área luminal mínima e diâmetro luminal foi baixa entre os dois métodos de imagem, com o coeficiente de correlação intraclasse variando de 0,11 a 0,52 (Tabela 7).

Tabela 7. Concordância das medidas quantitativas entre as imagens obtidas pela tomografia de coerência óptica e pela tomografia computadorizada de 320 detectores

\begin{tabular}{|c|c|c|c|}
\hline & OCT & TC320 & $\mathrm{CCl}(\mathrm{IC} \mathrm{95 \% )}$ \\
\hline \multicolumn{4}{|l|}{ Área do lúmen $\left(\mathrm{mm}^{2}\right)$} \\
\hline Média \pm DP & $2,78 \pm 1,23$ & $3,29 \pm 1,49$ & $0,52(0,26-0,71)$ \\
\hline Mínimo - Máximo & $0,89-5,48$ & $1,00-7,00$ & \\
\hline \multicolumn{4}{|c|}{ Diâmetro médio do lúmen (mm) } \\
\hline Média \pm DP & $2,71 \pm 0,47$ & $2,04 \pm 0,56$ & $0,16(-0,08-0,42)$ \\
\hline Mínimo - Máximo & $1,77-3,67$ & $0,50-3,30$ & \\
\hline \multicolumn{4}{|c|}{ Diâmetro mínimo do lúmen (mm) } \\
\hline Média \pm DP & $2,46 \pm 0,43$ & $1,85 \pm 0,55$ & $0,11(-0,09-0,33)$ \\
\hline Mínimo - Máximo & $1,61-3,32$ & $0,50-3,30$ & \\
\hline \multicolumn{4}{|c|}{ Diâmetro máximo do lúmen (mm) } \\
\hline Média \pm DP & $2,96 \pm 0,53$ & $2,24 \pm 0,69$ & $0,20(-0,08-0,47)$ \\
\hline Mínimo - Máximo & $1,94-4,12$ & $0,50-3,80$ & \\
\hline
\end{tabular}

CCI: Coeficiente de correlação intraclasse; . IC 95\%: Intervalo de confiança de 95\%; OCT: tomografia de coerência óptica; TC320: tomografia computadorizada de 320 detectores; DP: desvio-padrão. 
A análise dos gráficos de dispersão sugere que as imagens obtidas pela OCT fornecem valores superiores de diâmetros comparada às obtidas pela TC320 (Figura 4).
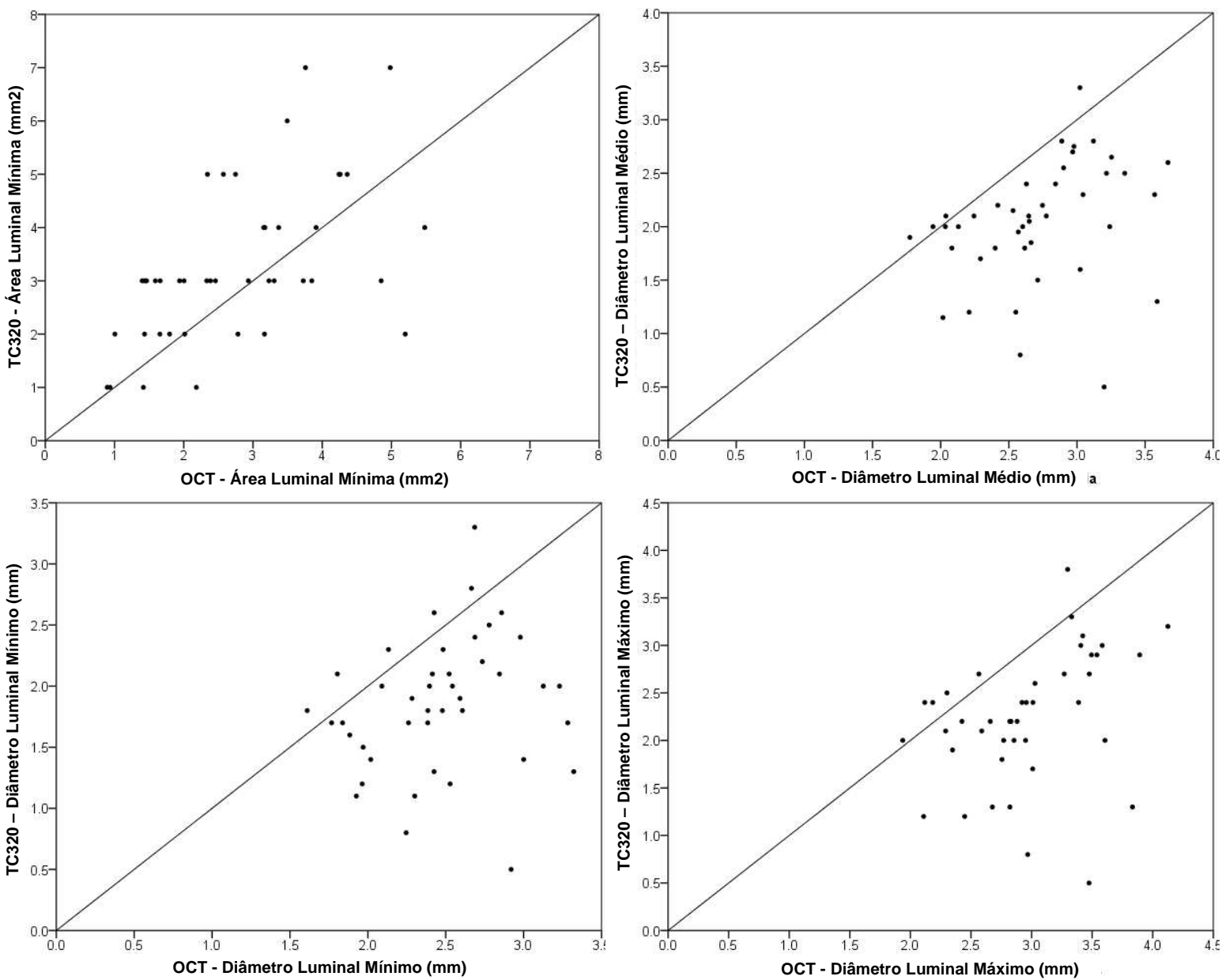

OCT: tomografia de coerência óptica; TC320: tomografia computadorizada de 320 detectores.

Figura 4. Diagramas de dispersão das medidas de área e de diâmetros entre as imagens obtidas pela tomografia de coerência óptica e pela tomografia computadorizada de 320 detectores 
Os gráficos de Bland-Altman sugerem, entretanto, não existir uma tendência da TC320 de super ou subestimar os valores obtidos pela OCT. A média de diferença entre as medidas de área luminal mínima, diâmetros luminais mínimo, máximo e médio foi de $0,50 \pm 1,29 \mathrm{~mm},-0,61 \pm 0,62 \mathrm{~mm}, 0,73 \pm 0,71$ e $0,67 \pm 0,62$, respectivamente (Figura 5).
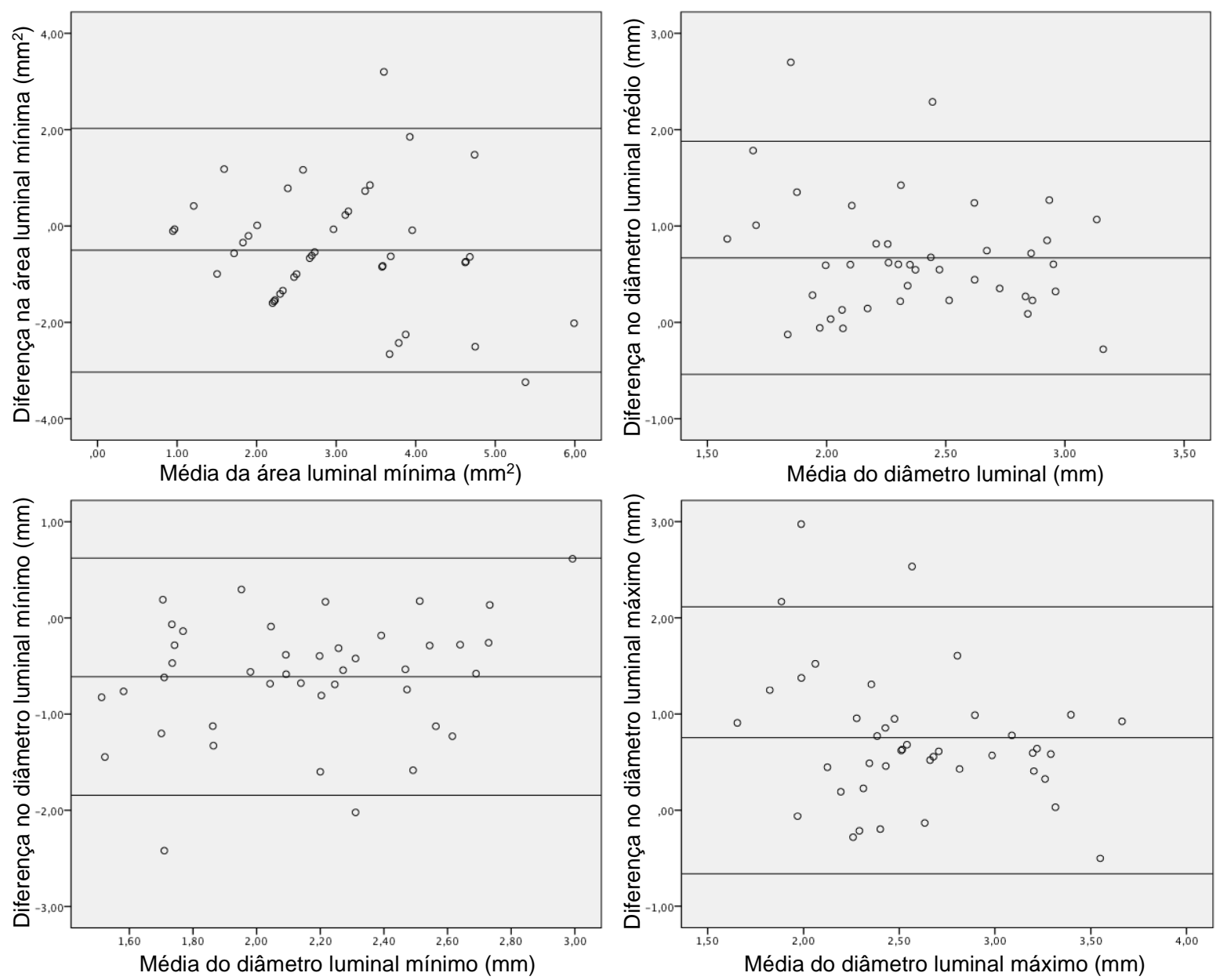

Figura 5. Gráficos de Bland-Altman das médias de diferenças e desvios padrões de área e de diâmetros entre as imagens obtidas pela tomografia de coerência óptica e pela tomografia computadorizada de 320 detectores

\subsubsection{Características de vulnerabilidade}

Avaliamos as medidas de acurácia das características de vulnerabilidade das imagens obtidas pela TC320 para a identificação dos fibroateromas, considerando as imagens obtidas pela OCT como padrão-ouro. As medidas indicaram que a melhor acurácia $(75,14 \%)$ pode ser obtida quando estão 
presentes concomitantemente a combinação de todas as características a seguir: sinal do anel de guardanapo (a), remodelamento positivo (b), presença de placa aterosclerótica com baixa atenuação (c) e de calcificação localizada (spotty calcification) (d) (Tabela 8).

Tabela 8. Medidas de acurácia das características de vulnerabilidade das imagens obtidas pela tomografia computadorizada de 320 detectores para a identificação de fibroateromas

\begin{tabular}{lccccc} 
& VP & FP & VN & FN & Acurácia (IC 95\%) \\
\hline Anel de guardanapo (a) & 8 & 14 & 18 & 2 & $60,91(44,3-77,5)$ \\
Remodelamento positivo (b) & 9 & 19 & 13 & 1 & $51,68(36,0-67,3)$ \\
Placa com baixa atenuação (c) & 6 & 10 & 22 & 4 & $69,04(56,1-82,0)$ \\
Calcificação localizada (d) & 4 & 12 & 20 & 6 & $56,11(40,1-72,1)$ \\
(a) ou (b) ou (c) ou (d) & 9 & 20 & 12 & 1 & $48,99(33,3-64,7)$ \\
(a) e (b) e (c) e (d) & 2 & 4 & 28 & 8 & $75,14(60,8-89,5)$ \\
(a) e (b) & 8 & 13 & 19 & 2 & $63,94(47,6-80,3)$ \\
(a) e (c) & 9 & 20 & 12 & 1 & $48,99(33,3-64,7)$ \\
(a) e (d) & 5 & 9 & 23 & 5 & $71,33(57,2-85,4)$ \\
(b) e (c) & 9 & 15 & 17 & 1 & $59,87(43,6-76,2)$ \\
(b) e (d) & 4 & 8 & 24 & 6 & $65,69(49,9-81,4)$ \\
(c) e (d) & 8 & 18 & 14 & 2 & $50,92(34,3-67,6)$ \\
(a) ou (b) & 6 & 9 & 23 & 4 & $71,17(58,6-83,8)$ \\
(a) ou (c) & 9 & 20 & 12 & 1 & $48,99(33,3-64,7)$ \\
(a) ou (d) & 4 & 11 & 21 & 6 & $58,83(43,0-74,7)$ \\
(b) ou (c) & 9 & 20 & 12 & 1 & $48,99(33,3-64,7)$ \\
(b) ou (d) & 2 & 6 & 26 & 8 & $71,33(57,2-85,4)$ \\
(c) ou (d) & 8 & 16 & 16 & 2 & $56,57(41,3-71,9)$ \\
(a) e (b) e (c) & 5 & 8 & 24 & 5 & $73,27(59,1-87,4)$ \\
(a) e (b) e (d) & 9 & 20 & 12 & 1 & $48,99(33,3-64,7)$ \\
(a) e (c) e (d) & 4 & 7 & 25 & 6 & $68,58(53,2-84,0)$ \\
(b) e (c) e (d) & 9 & 20 & 12 & 1 & $48,99(33,3-64,7)$ \\
(a) ou (b) ou (c) & 2 & 5 & 27 & 8 & $73,27(59,1-87,4)$ \\
(a) ou (b) ou (d) & 9 & 18 & 14 & 1 & $53,33(37,2-69,4)$ \\
(a) ou (c) ou (d) & 2 & 5 & 27 & 8 & $73,27(59,1-87,4)$ \\
(b) ou (c) ou (d) & 9 & 20 & 12 & 1 & $48,99(33,3-64,7)$ \\
\hline n & 92 VP: vra &
\end{tabular}

n=42; VP: verdadeiros positivos; FP: falsos positivos; VN: verdadeiros negativos; FN: falsos negativos; IC95\%: intervalo de confiança de 95\%.

Dentre as quatro características associadas à vulnerabilidade das placas ateroscleróticas, o sinal de anel de guardanapo foi o único que se mostrou associado a piores fenótipos $(p=0,036)$.

A presença de pelo menos 2 sinais de vulnerabilidade das placas ateroscleróticas nas imagens obtidas pela TC320 foi associada a maior quantidade de macrófagos $(p=0,034)$ e cristais de colesterol $(p=0,04)$ presentes nas imagens obtidas pela OCT (Tabela 9). O sinal de anel do guardanapo visto nas imagens 
obtidas pela TC320 foi o único componente que isoladamente foi associado à presença de macrófagos nas imagens obtidas pela OCT $(p=0,008)$.

Tabela 9. Associação de sinais de vulnerabilidade da placa aterosclerótica das imagens obtidas pela tomografia computadorizada de 320 detectores e características de vulnerabilidade das imagens obtidas pela tomografia de coerência óptica

\begin{tabular}{lccc}
\hline \multirow{2}{*}{ OCT } & \multicolumn{3}{c}{ TC320 } \\
\cline { 2 - 4 } & $\begin{array}{c}\text { Sinais de } \\
\text { vulnerabilidade }\end{array}$ & $\begin{array}{c}\text { Ausência de sinais de } \\
\text { vulnerabilidade }\end{array}$ & $\begin{array}{c}\text { Valor- } \\
\mathbf{p}\end{array}$ \\
\hline Angulo do Arco Lipídico (graus) & 9 & 1 & --- \\
$\quad$ Mediana (10 quartil - 3ํquartil) & $223,4(151,2-317,9)$ & 183,5 & \\
$\quad$ Mínimo - Máximo & $97,6-360,0$ & & \\
Macrófago & $23(74,2 \%)$ & $8(25,8 \%)$ & 0,034 \\
Neovascularização & $3(50,0 \%)$ & $3(50,0 \%)$ & 0,649 \\
Cristais de colesterol & $12(85,7 \%)$ & $2(14,3 \%)$ & 0,040 \\
\hline Valores apresentados em números & absolutos e frequência; Legenda: OCT: tomografia de \\
coerência óptica; TC320: tomografia computadorizada de 320 detectores. &
\end{tabular}

\subsubsection{Fibroateroma versus não-fibroateroma}

Ao classificarmos as placas ateroscleróticas observadas nas imagens obtidas pela OCT em fibroateroma e não-fibroateroma, observamos que a mediana de excentricidade do lúmen dos não-fibroateromas $(0,16$; IC 95\% 0,14 $0,19)$ foi maior do que a dos fibroateromas $(0,20$; IC $95 \% 0,16-0,21 ; p=0,045)$. Macrófagos foram vistos em todos os fibroateromas e em $65,6 \%$ dos nãofibroateromas $(p=0,041)$ (Tabela 10).

Houve uma tendência de maior presença do sinal do anel de guardanapo $(80,0 \%$ versus $43,8 \% ; p=0,071)$ e de características de vulnerabilidade da placa aterosclerótica $(90,0 \%$ versus $56,3 \% ; p=0,068)$ nas imagens obtidas pela TC320 nos fibroateromas comparados aos não-fibroateromas (Tabela 10). Não houve associação entre o tipo de placa aterosclerótica pela TC320 e a presença ou não de fibroateroma $(p=0,615)$. 
Tabela 10. Associação das variáveis observadas à tomografia computadorizada de 320 detectores e à tomografia de coerência óptica com o fibroateroma definido pela tomografia de coerência óptica

\begin{tabular}{|c|c|c|c|c|c|c|}
\hline & \multicolumn{2}{|c|}{ TC320 } & \multicolumn{3}{|c|}{ OCT } & \multirow[b]{2}{*}{ Valor-p } \\
\hline & $\begin{array}{c}\text { Não-fibroateroma } \\
(\mathrm{N}=32)\end{array}$ & $\begin{array}{c}\text { Fibroateroma } \\
(N=10)\end{array}$ & Valor-p & $\begin{array}{c}\text { Não-fibroateroma } \\
(\mathrm{N}=32)\end{array}$ & $\begin{array}{l}\text { Fibroateroma } \\
\qquad(\mathrm{N}=10)\end{array}$ & \\
\hline $\begin{array}{l}\text { Área luminal mínima } \\
\left(\mathrm{mm}^{2}\right)\end{array}$ & $3,00(2,00-4,00)$ & $3,00(2,00-4,00)$ & 0,64 & $2,81 \pm 1,33$ & $2,71 \pm 0,90$ & 0,78 \\
\hline $\begin{array}{l}\text { Diâmetro luminal médio } \\
(\mathrm{mm})\end{array}$ & $2,10 \pm 0,49$ & $1,85 \pm 0,75$ & 0,35 & $2,70 \pm 0,49$ & $2,72 \pm 0,45$ & 0,91 \\
\hline $\begin{array}{l}\text { Diâmetro luminal mínimo } \\
(\mathrm{mm})\end{array}$ & $1,90 \pm 0,51$ & $1,68 \pm 0,65$ & 0,26 & $2,47 \pm 0,45$ & $2,45 \pm 0,40$ & 0,90 \\
\hline $\begin{array}{l}\text { Diâmetro luminal máximo } \\
(\mathrm{mm})\end{array}$ & $2,30 \pm 0,55$ & $2,03 \pm 1,03$ & 0,44 & $2,94 \pm 0,54$ & $3,02 \pm 0,51$ & 0,70 \\
\hline $\begin{array}{l}\text { Indice excentricidade } \\
\text { luminal }\end{array}$ & & & & $0,16(0,14-0,19)$ & $0,20(0,16-0,21)$ & 0,045 \\
\hline Assimetria luminal & & & & $0,42(0,36-0,47)$ & $0,43(0,32-0,45)$ & 0,62 \\
\hline $\begin{array}{l}\text { Estenose luminal } \\
\text { Estenose, }\end{array}$ & & & & $65,69(55,77-72,98)$ & $60,51(51,80-74,43)$ & 0,72 \\
\hline Normal & $0(0,0 \%)$ & $0(0,0 \%)$ & $>0,99$ & $0(0,0 \%)$ & $0(0,0 \%)$ & $>0,99$ \\
\hline Mínima (0-24\%) & $1(3,1 \%)$ & $0(0,0 \%)$ & & $2(6,3 \%)$ & $0(0,0 \%)$ & \\
\hline Leve (25-49\%) & $8(25,0 \%)$ & $2(20,0 \%)$ & & $3(9,4 \%)$ & $1(10,0 \%)$ & \\
\hline Moderada (50-69\%) & $12(37,5 \%)$ & $4(40,0 \%)$ & & $17(53,1 \%)$ & $6(60,0 \%)$ & \\
\hline Grave ( $\geq 70 \%)$ & $11(34,4 \%)$ & $4(40,0 \%)$ & & $10(31,3 \%)$ & $3(30,0 \%)$ & \\
\hline Macrófago & & & & $21(65,6 \%)$ & $10(100,0 \%)$ & 0,041 \\
\hline Neovascularização & & & & $5(15,6 \%)$ & $1(10,0 \%)$ & $>0,99$ \\
\hline Cristais de Colesterol & & & & $9(28,1 \%)$ & $5(50,0 \%)$ & 0,26 \\
\hline $\begin{array}{l}\text { Placa vulnerável (definição } \\
\text { CAD-RADS) }\end{array}$ & $18(56,3 \%)$ & $9(90,0 \%)$ & 0,068 & & & \\
\hline $\begin{array}{l}\text { Sinal do anel de } \\
\text { quardanapo }\end{array}$ & $14(43,8 \%)$ & $8(80,0 \%)$ & 0,071 & & & \\
\hline Remodelamento positivo & $19(59,4 \%)$ & $9(90,0 \%)$ & 0,13 & & & \\
\hline $\begin{array}{l}\text { Placa com baixa } \\
\text { atenuacão }(<30 \mathrm{HU})\end{array}$ & $10(31,3 \%)$ & $6(60,0 \%)$ & 0,14 & & & \\
\hline $\begin{array}{l}\text { Calcificação localizada } \\
\text { Tipo de placa } \\
\text { aterosclerótica }\end{array}$ & $12(37,5 \%)$ & $4(40,0 \%)$ & $>0,99$ & & & \\
\hline Normal & $0(0,0 \%)$ & $0(0,0 \%)$ & 0,62 & & & \\
\hline Calcificada & $5(15,6 \%)$ & $1(10,0 \%)$ & & & & \\
\hline Mista & $13(40,6 \%)$ & $6(60,0 \%)$ & & & & \\
\hline Não calcificada & $14(43,8 \%)$ & $3(30,0 \%)$ & & & & \\
\hline
\end{tabular}

Valores apresentados em números absolutos e frequência, média e desvio padrão e mediana e intervalo interquartil; Legenda: OCT: tomografia de coerência óptica; TC320: tomografia computadorizada de 320 detectores. 


\section{DISCUSSÃO}

Os resultados deste estudo sugerem que as imagens obtidas pela angioTC coronária realizada com a TC320 foi capaz de diferenciar as placas ateroscleróticas calcificadas e não calcificadas identificadas nas imagens obtidas pela OCT. Entretanto, dentre as placas ateroscleróticas não calcificadas, a TC320 não foi capaz de diferenciar quanto à presença ou ausência de fibroateroma.

A correlação e a concordância dos tipos de placas ateroscleróticas normalmente descritos nas imagens obtidas pela OCT (calcificada, fibrótica e fibroateroma) e pela angioTC (calcificada, mista e não calcificada) não são possíveis por descreverem componentes diferentes da placa aterosclerótica. Placas ateroscleróticas consideradas não calcificadas nas imagens obtidas pela angioTC podem ser classificadas tanto em placas aterosclerótica fibróticas quanto em fibroateromas nas imagens obtidas pela OCT. O mesmo pode ocorrer com as placas ateroscleróticas mistas vistas nas imagens obtidas pela angioTC, dependendo do achado ou não do componente de baixa atenuação.

Estudos prévios encontraram entre $30 \%$ e $35,6 \%$ de placas ateroscleróticas fibróticas vistos nas imagens obtidas pela OCT nas placas aterosclerótica não calcificadas das imagens obtidas pela angioTC. ${ }^{(62,65)} \mathrm{Em}$ nosso estudo, identificamos cerca de $70 \%$ de placas ateroscleróticas fibróticas entre as não placas ateroscleróticas calcificadas. Esta diferença pode ser secundária à população de cada estudo; naqueles, foram incluídos apenas pacientes com SCA, contexto relacionado a uma presença maior de fibroateromas do que placas ateroscleróticas fibróticas.

Petranovic et al. ${ }^{(71)}$ demonstraram que as análises das imagens obtidas na angioTC podem superestimar os valores de área do vaso comparada ao ultrassom intra-coronário. Por outro lado, Yang et al. ${ }^{(67)}$ mostraram que as análises das imagens obtidas pela angioTC superestimam discretamente a área luminal medida nas imagens obtidas pela OCT. Nossos resultados mostram que a análise das imagens obtidas pela TC320 subestimaram os valores de diâmetro luminal quando comparada às imagens obtidas pela OCT. A baixa concordância nas medidas de diâmetro e área luminal para quantificação de estenoses coronárias entre as imagens obtidas pela angioTC, pela cinecoronariografia, pelo ultrassom intracoronário e pela OCT é comum 
e pode ocorrer devido a diversas razões como a qualidade da imagem, a experiência limitada do operador, a variabilidade na seleção do local de referência e a inferior resolução espacial da angioTC. ${ }^{(72)}$

Desta maneira, a Sociedade de Tomografia Computadorizada Cardiovascular propôs uma classificação graduando as estenoses coronárias de maneira semi-quantitativa e categorizando seus resultados, como o proposto pelo sistema CAD-RADS. ${ }^{(70)}$ Apesar da concordância desta metodologia ter se mostrado alta em nosso estudo, sua acurácia foi baixa $(42,1 \%),(A C=0,64)$. A concordância perfeita do grau de estenose entre as imagens obtidas pela angioTC e pela OCT é não apenas irreal como também de relevância incerta para a ocorrência de desfechos clínicos. A avaliação do tipo de placa aterosclerótica (calcificada e não calcificada), da carga de placa aterosclerótica, do número e do local das lesões, assim como detalhes da caracterização da placa aterosclerótica podem ter um impacto superior à sua simples quantificação.

A vulnerabilidade da placa aterosclerótica, de acordo com o sistema CAD-RADS, ocorre quando pelo menos duas das seguintes características estão presentes: placa aterosclerótica de baixa atenuação $(<30 \mathrm{HU})$, remodelamento positivo, sinal do anel de guardanapo e calcificação localizada. Estudos prévios consistentemente demonstraram que a placa aterosclerótica de baixa atenuação e o sinal de anel de guardanapo são associados a presença de fibroateroma de capa fina. ${ }^{(66,67,73)}$ Em nosso estudo, estas variáveis foram as que demonstraram maior acurácia para a detecção do fibroateroma $(69 \%$ e $61 \%$, respectivamente). A calcificação localizada ou puntiforme é descrita como um ponto de cálcio dentro da placa aterosclerótica. Acreditamos haver espaço para uma definição mais especifica desta característica, de modo que ela não se confunda com uma placa aterosclerótica calcificada, por exemplo. Estudos anteriores mostraram resultados inconsistentes quanto à relação da calcificação localizada e da placa aterosclerótica vulnerável, ${ }^{(67,74-76)}$ sendo que em outras publicações este parâmetro tampouco foi incluído nas análises metodológicas.

O agrupamento de características presentes nas imagens dos métodos descritos pode levar a uma maior acurácia na identificação do fibroateroma. Em nosso estudo a associação de todas as quatro variáveis levou uma acurácia superior $(75 \%)$ àquelas observadas pelas outras possíveis combinações do sistema CAD-RADS. Soeda el al. ${ }^{(62)}$ agruparam as variáveis área transversal de núcleo lipídico 
$>2,7 \mathrm{~mm}^{2}$, a placa aterosclerótica com atenuação $<50 \mathrm{HU}$ e calcificação concomitante e encontraram uma acurácia de $67,1 \%$. A presença de área transversal de núcleo lipídico $>2,7 \mathrm{~mm}^{2}$, placa aterosclerótica com atenuação $<50 \mathrm{HU}$ ou calcificação concomitante levou a uma acurácia de 60,5\%. Mais recentemente, Tomizawa et al. ${ }^{(66)}$ propuseram três modelos para identificação do fibroteroma de capa fina: o modelo 1 se baseava no índice de remodelamento positivo $>1,1$, atenuação $<30 \mathrm{HU}$ e presença do sinal do anel de guardanapo; o modelo 2 incluía índice de remodelamento positivo $>1,1$, atenuação $<30 \mathrm{HU}$ ou presença do sinal do anel de guardanapo; e o modelo 3 utilizava uma análise de regressão logística que incluía o índice de remodelamento, a proporção do volume de baixa atenuação $(<60 \mathrm{HU})$ e a presença do sinal de anel de guardanapo. $O$ modelo 3 mostrou uma área sob a curva de 0,96, significativamente superior aos dos demais modelos, com uma acurácia de $93 \%$, sugerindo que o uso das variáveis do índice de remodelamento e da proporção do volume de baixa atenuação de forma contínua seja superior que sua dicotomização.

Alguns atributos observados nas imagens obtidas pela OCT sugerem aspectos de vulnerabilidade da placa aterosclerótica, como a presença de neovascularização, de cristais de colesterol e, especialmente, de macrófagos. ${ }^{(77)} \mathrm{A}$ infiltração de macrófagos pode enfraquecer a integridade estrutural da capa fibrótica e aumentar sua chance de ruptura. Como verificado em nosso estudo, é possível que a presença de maior vulnerabilidade, de acordo com o sistema CAD-RADS, presente nas imagens obtidas pela angioTC esteja associada à algumas características observadas nas imagens obtidas pela OCT, reforçando o conjunto de parâmetros propostos pelo CAD-RADS para se encontrar placas ateroscleróticas vulneráveis.

Este estudo apresenta algumas limitações. Embora as análises das imagens tenham sido feitas prospectivamente, trata-se de um estudo retrospectivo observacional, sujeito às limitações deste tipo de análise. Apesar da análise histopatológica, padrão-ouro para definição do tipo de placa aterosclerótica, não ter sido realizada, já foi demonstrado que as imagens obtidas pela OCT possuem grande acurácia na definição dos componentes e da graduação da lesão da placa aterosclerótica quando comparadas àquelas observadas à histopatologia. ${ }^{(64)}$ As imagens dos segmentos coronários analisadas foram obtidas de uma amostra pequena com diferentes apresentações clinicas, o que limita a validade interna do estudo, contudo expandindo sua generalibidade. Não foram observados fibroateromas de capa 
fina em nossa amostra. Mais investigações são necessárias para a validação de nossos resultados dentro deste subtipo específico de placa aterosclerótica.

Este é o primeiro estudo a comparar resultados das análises das imagens obtidas pela angioTC realizada na TC320 com as imagens obtidas pela OCT. Apesar de possuir uma maior resolução temporal, as imagens obtidas pela TC320 não foram capazes de diferenciar os fibroateromas identificados nas imagens obtidas pela OCT. Como perspectivas futuras, acreditamos que a evolução tecnológica deva focar também em equipamentos com maior resolução espacial, assim como em definições mais especificas dos possíveis componentes das placas ateroscleróticas vulneráveis. A identificação de maneira não-invasiva das placas ateroscleróticas com maior propensão para causar SCA continuará sendo uma das principais linhas de pesquisa dentro da cardiologia moderna por possibilitar uma possível intervenção precoce nestes pacientes 


\section{CONCLUSÕES}

1. A análise das imagens obtidas pela tomografia computadorizada de 320 detectores foi capaz de diferenciar as placas ateroscleróticas calcificadas das não calcificadas observadas nas imagens obtidas pela tomografia de coerência óptica.

2. A análise das imagens obtidas pela tomografia computadorizada de 320 detectores não foi capaz de diferenciar a presença dos fibroateromas identificados nas imagens obtidas pela tomografia de coerência óptica.

3. A associação das características tomográficas do sinal do anel de guardanapo, do remodelamento positivo, da placa aterosclerótica de baixa atenuação e da calcificação localizada apresentou a maior acurácia para a identificação do fibroateroma.

4. A concordância das medidas de diâmetro e de área dos segmentos coronarianos entre as imagens obtidas pela tomografia computadorizada de 320 detectores e pela tomografia de coerência óptica foram baixas. 


\section{REFERÊNCIAS}

1. Faxon DP, Fuster V, Libby P, Beckman JA, Hiatt WR, Thompson RW, et al. Atherosclerotic Vascular Disease Conference: Writing Group III: pathophysiology. Circulation. 2004;109(21):2617-25.

2. Libby $P$, Ridker PM, Hansson GK. Progress and challenges in translating the biology of atherosclerosis. Nature. 2011;473(7347):317-25.

3. Nam CW, Yoon HJ, Cho YK, Park HS, Kim H, Hur SH, et al. Outcomes of percutaneous coronary intervention in intermediate coronary artery disease: fractional flow reserve-guided versus intravascular ultrasound-guided. JACC Cardiovasc Interv. 2010;3(8):812-7.

4. Toth GG, Toth B, Johnson NP, De Vroey F, Di Serafino L, Pyxaras S, et al. Revascularization Decisions in Patients With Stable Angina and Intermediate Lesions: Results of the International Survey on Interventional Strategy. Circ Cardiovasc Interv. 2014;7(6):751-9.

5. Hoffmann U, Moselewski F, Nieman K, Jang IK, Ferencik M, Rahman AM, et al. Noninvasive assessment of plaque morphology and composition in culprit and stable lesions in acute coronary syndrome and stable lesions in stable angina by multidetector computed tomography. J Am Coll Cardiol. 2006;47(8):1655-62.

6. Fujii K, Kobayashi Y, Mintz GS, Takebayashi H, Dangas G, Moussa I, et al. Intravascular ultrasound assessment of ulcerated ruptured plaques: a comparison of culprit and nonculprit lesions of patients with acute coronary syndromes and lesions in patients without acute coronary syndromes. Circulation. 2003;108(20):2473-8.

7. Pundziute G, Schuijf JD, Jukema JW, Decramer I, Sarno G, Vanhoenacker PK, et al. Evaluation of plaque characteristics in acute coronary syndromes: non-invasive assessment with multi-slice computed tomography and invasive evaluation with intravascular ultrasound radiofrequency data analysis. Eur Heart J. 2008;29(19):2373-81.

8. Task Force Members, Montalescot G, Sechtem U, Achenbach S, Andreotti F, Arden C, Budaj A, Bugiardini R, Crea F, Cuisset T, Di Mario C, Ferreira JR, Gersh BJ, Gitt AK, Hulot JS, Marx N, Opie LH, Pfisterer M, Prescott E, Ruschitzka F, Sabaté M, Senior R, Taggart DP, van der Wall EE, Vrints CJ; ESC Committee for Practice Guidelines, Zamorano JL, Achenbach S, Baumgartner H, Bax JJ, Bueno H, Dean V, Deaton C, Erol C, Fagard R, Ferrari R, Hasdai D, Hoes AW, Kirchhof P, Knuuti J, Kolh P, Lancellotti P, Linhart A, Nihoyannopoulos P, Piepoli MF, Ponikowski P, Sirnes PA, Tamargo JL, Tendera M, Torbicki A, Wijns W, Windecker S;

Document Reviewers, Knuuti J, Valgimigli M, Bueno H, Claeys MJ, Donner-Banzhoff N, Erol C, Frank H, Funck-Brentano C, Gaemperli O, Gonzalez-Juanatey JR, Hamilos M, Hasdai D, Husted S, James SK, Kervinen K, Kolh P, Kristensen SD, Lancellotti P, Maggioni AP, Piepoli MF, Pries AR, Romeo F, Rydén L, Simoons ML, Sirnes PA, Steg PG, Timmis A, Wijns W, Windecker S, Yildirir A, Zamorano JL. 2013 ESC guidelines on the management of stable coronary artery disease: the Task Force on the management of stable coronary artery disease of the European Society of Cardiology. Eur Heart J. 2013;34(38):2949-3003. 
9. Stary HC, Chandler AB, Dinsmore RE, Fuster V, Glagov S, Insull W, Jr., et al. A definition of advanced types of atherosclerotic lesions and a histological classification of atherosclerosis. $A$ report from the Committee on Vascular Lesions of the Council on Arteriosclerosis, American Heart Association. Circulation. 1995;92(5):1355-74.

10. Tuzcu EM, Kapadia SR, Tutar E, Ziada KM, Hobbs RE, McCarthy PM, et al. High prevalence of coronary atherosclerosis in asymptomatic teenagers and young adults: evidence from intravascular ultrasound. Circulation. 2001;103(22):2705-10.

11. Strong JP, Malcom GT, McMahan CA, Tracy RE, Newman WP 3rd, Herderick EE, et al. Prevalence and extent of atherosclerosis in adolescents and young adults: implications for prevention from the Pathobiological Determinants of Atherosclerosis in Youth Study. JAMA. 1999;281(8):727-35.

12. Kitta Y, Obata JE, Nakamura T, Hirano M, Kodama Y, Fujioka D, et al. Persistent impairment of endothelial vasomotor function has a negative impact on outcome in patients with coronary artery disease. J Am Coll Cardiol. 2009;53(4):323-30.

13. Gordon T, Castelli WP, Hjortland MC, Kannel WB, Dawber TR. High density lipoprotein as a protective factor against coronary heart disease. The Framingham Study. Am J Med. 1977;62(5):707-14.

14. Criqui MH, Heiss G, Cohn R, Cowan LD, Suchindran CM, Bangdiwala S, et al. Plasma triglyceride level and mortality from coronary heart disease. N Engl J Med. 1993;328(17):12205 .

15. Romm PA, Green CE, Reagan K, Rackley CE. Relation of serum lipoprotein cholesterol levels to presence and severity of angiographic coronary artery disease. Am J Cardiol. 1991;67(6):479-83.

16. Iuliano L, Mauriello A, Sbarigia E, Spagnoli LG, Violi F. Radiolabeled native low-density lipoprotein injected into patients with carotid stenosis accumulates in macrophages of atherosclerotic plaque : effect of vitamin E supplementation. Circulation. 2000;101(11):1249-54.

17. Tabas I. Consequences of cellular cholesterol accumulation: basic concepts and physiological implications. J Clinl Invest. 2002;110(7):905-11.

18. Hansson GK. Inflammation, atherosclerosis, and coronary artery disease. N Engl J Med. 2005;352(16):1685-95.

19. Weber C, Noels $\mathrm{H}$. Atherosclerosis: current pathogenesis and therapeutic options. Nat Med. 2011;17(11):1410-22. 
20. Davies MJ, Woolf N, Rowles PM, Pepper J. Morphology of the endothelium over atherosclerotic plaques in human coronary arteries. Br Heart J. 1988;60(6):459-64.

21. Sata M, Saiura A, Kunisato A, Tojo A, Okada S, Tokuhisa T, et al. Hematopoietic stem cells differentiate into vascular cells that participate in the pathogenesis of atherosclerosis. Nat Med. 2002;8(4):403-9.

22. Kockx MM, De Meyer GR, Muhring J, Jacob W, Bult H, Herman AG. Apoptosis and related proteins in different stages of human atherosclerotic plaques. Circulation. 1998;97(23):2307-15.

23. Heistad DD, Marcus ML, Larsen GE, Armstrong ML. Role of vasa vasorum in nourishment of the aortic wall. Am J Physiol. 1981;240(5):H781-7.

24. Barger AC, Beeuwkes R 3rd, Lainey LL, Silverman KJ. Hypothesis: vasa vasorum and neovascularization of human coronary arteries. A possible role in the pathophysiology of atherosclerosis. N Engl J Med. 1984;310(3):175-7.

25. Kolodgie FD, Gold HK, Burke AP, Fowler DR, Kruth HS, Weber DK, et al. Intraplaque hemorrhage and progression of coronary atheroma. N Engl J Med. 2003;349(24):2316-25.

26. Schoenhagen P, Ziada KM, Vince DG, Nissen SE, Tuzcu EM. Arterial remodeling and coronary artery disease: the concept of "dilated" versus "obstructive" coronary atherosclerosis. J Am Coll Cardiol. 2001;38(2):297-306.

27. Schoenhagen P, Ziada KM, Kapadia SR, Crowe TD, Nissen SE, Tuzcu EM. Extent and direction of arterial remodeling in stable versus unstable coronary syndromes: an intravascular ultrasound study. Circulation. 2000;101(6):598-603.

28. Falk E, Shah PK, Fuster V. Coronary plaque disruption. Circulation. 1995;92(3):657-71.

29. Ambrose JA, Tannenbaum MA, Alexopoulos D, Hjemdahl-Monsen CE, Leavy J, Weiss M, et al. Angiographic progression of coronary artery disease and the development of myocardial infarction. J Am Coll Cardiol. 1988;12(1):56-62.

30. Kubo T, Imanishi T, Takarada S, Kuroi A, Ueno S, Yamano T, et al. Assessment of culprit lesion morphology in acute myocardial infarction: ability of optical coherence tomography compared with intravascular ultrasound and coronary angioscopy. J Am Coll Cardiol. 2007;50(10):933-9.

31. Burke AP, Kolodgie FD, Farb A, Weber DK, Malcom GT, Smialek J, et al. Healed plaque ruptures and sudden coronary death: evidence that subclinical rupture has a role in plaque progression. Circulation. 2001;103(7):934-40. 
32. Mintz GS, Nissen SE, Anderson WD, Bailey SR, Erbel R, Fitzgerald PJ, et al. American College of Cardiology Clinical Expert Consensus Document on Standards for Acquisition, Measurement and Reporting of Intravascular Ultrasound Studies (IVUS). A report of the American College of Cardiology Task Force on Clinical Expert Consensus Documents. J Am Coll Cardiol. 2001;37(5):1478-92.

33. Prati F, Regar E, Mintz GS, Arbustini E, Di Mario C, Jang IK, Akasaka T, Costa M, Guagliumi G, Grube E, Ozaki Y, Pinto F, Serruys PW; Expert's OCT Review Document. Expert review document on methodology, terminology, and clinical applications of optical coherence tomography: physical principles, methodology of image acquisition, and clinical application for assessment of coronary arteries and atherosclerosis. Eur Heart J. 2010;31(4):401-15.

34. Huang D, Swanson EA, Lin CP, Schuman JS, Stinson WG, Chang W, et al. Optical coherence tomography. Science. 1991;254(5035):1178-81.

35. Tearney GJ, Waxman S, Shishkov M, Vakoc BJ, Suter MJ, Freilich MI, et al. Threedimensional coronary artery microscopy by intracoronary optical frequency domain imaging. JACC Cardiovasc Imaging. 2008;1(6):752-61.

36. Davlouros P, Damelou A, Karantalis V, Xanthopoulou I, Mavronasiou E, Tsigkas G, et al. Evaluation of culprit saphenous vein graft lesions with optical coherence tomography in patients with acute coronary syndromes. JACC Cardiovasc Interv 2011;4(6):683-93.

37. Imola F, Mallus MT, Ramazzotti V, Manzoli A, Pappalardo A, Di Giorgio A, et al. Safety and feasibility of frequency domain optical coherence tomography to guide decision making in percutaneous coronary intervention. Eurolntervention. 2010;6(5):575-81.

38. Kawasaki M, Bouma BE, Bressner J, Houser SL, Nadkarni SK, MacNeill BD, et al. Diagnostic accuracy of optical coherence tomography and integrated backscatter intravascular ultrasound images for tissue characterization of human coronary plaques. J Am Coll Cardiol. 2006;48(1):81-8.

39. Yabushita H, Bouma BE, Houser SL, Aretz HT, Jang IK, Schlendorf KH, et al. Characterization of human atherosclerosis by optical coherence tomography. Circulation. 2002;106(13):1640-5.

40. Gonzalo N, Tearney GJ, Serruys PW, van Soest G, Okamura T, García-García HM, et al. Second-generation optical coherence tomography in clinical practice. High-speed data acquisition is highly reproducible in patients undergoing percutaneous coronary intervention. Rev Esp Cardiol. 2010;63(8):893-903.

41. Low AF, Kawase Y, Chan YH, Tearney GJ, Bouma BE, Jang IK. In vivo characterisation of coronary plaques with conventional grey-scale intravascular ultrasound: correlation with optical coherence tomography. Eurolntervention. 2009;4(5):626-32. 
42. Kume T, Okura H, Yamada R, Kawamoto T, Watanabe N, Neishi Y, et al. Frequency and spatial distribution of thin-cap fibroatheroma assessed by 3-vessel intravascular ultrasound and optical coherence tomography: an ex vivo validation and an initial in vivo feasibility study. Circ J. 2009;73(6):1086-91.

43. Cilingiroglu M, Oh JH, Sugunan B, Kemp NJ, Kim J, Lee S, et al. Detection of vulnerable plaque in a murine model of atherosclerosis with optical coherence tomography. Catheter Cardiovasc Interv. 2006;67(6):915-23.

44. Tearney GJ, Yabushita H, Houser SL, Aretz HT, Jang IK, Schlendorf KH, et al. Quantification of macrophage content in atherosclerotic plaques by optical coherence tomography. Circulation. 2003;107(1):113-9.

45. Tearney GJ, Regar E, Akasaka T, Adriaenssens T, Barlis P, Bezerra HG, Bouma B, Bruining N, Cho JM, Chowdhary S, Costa MA, de Silva R, Dijkstra J, Di Mario C, Dudek D, Falk E, Feldman MD, Fitzgerald P, Garcia-Garcia HM, Gonzalo N, Granada JF, Guagliumi G, Holm NR, Honda Y, Ikeno F, Kawasaki M, Kochman J, Koltowski L, Kubo T, Kume T, Kyono H, Lam CC, Lamouche G, Lee DP, Leon MB, Maehara A, Manfrini O, Mintz GS, Mizuno K, Morel MA, Nadkarni S, Okura H, Otake H, Pietrasik A, Prati F, Räber L, Radu MD, Rieber J, Riga M, Rollins A, Rosenberg M, Sirbu V, Serruys PW, Shimada K, Shinke T, Shite J, Siegel E, Sonoda S, Suter M, Takarada S, Tanaka A, Terashima M, Thim T, Uemura S, Ughi GJ, van Beusekom $H M$, van der Steen AF, van Es GA, van Soest G, Virmani R, Waxman S, Weissman NJ, Weisz G; International Working Group for Intravascular Optical Coherence Tomography (IWG-IVOCT). Consensus standards for acquisition, measurement, and reporting of intravascular optical coherence tomography studies: a report from the International Working Group for Intravascular Optical Coherence Tomography Standardization and Validation. J Am Coll Cardiol. 2012;59(12):1058-72.

46. Regar E, van Beusekom HM, van der Giessen WJ, Serruys PW. Images in cardiovascular medicine. Optical coherence tomography findings at 5-year follow-up after coronary stent implantation. Circulation. 2005;112(23):e345-6.

47. Raff GL, Gallagher MJ, O'Neill WW, Goldstein JA. Diagnostic accuracy of noninvasive coronary angiography using 64-slice spiral computed tomography. J Am Coll Cardiol. 2005;46(3):552-7.

48. Mollet NR, Cademartiri F, van Mieghem CA, Runza G, McFadden EP, Baks T, et al. Highresolution spiral computed tomography coronary angiography in patients referred for diagnostic conventional coronary angiography. Circulation. 2005;112(15):2318-23.

49. Hsiao EM, Rybicki FJ, Steigner M. CT coronary angiography: 256-slice and 320-detector row scanners. Curr Cardiol Rep. 2010;12(1):68-75.

50. Hurlock GS, Higashino H, Mochizuki T. History of cardiac computed tomography: single to 320-detector row multislice computed tomography. Int J Cardiovasc Imaging. 2009;25 Suppl $1: 31-42$. 
51. Einstein AJ, Elliston CD, Arai AE, Chen MY, Mather R, Pearson GD, et al. Radiation dose from single-heartbeat coronary CT angiography performed with a 320-detector row volume scanner. Radiology. 2010;254(3):698-706.

52. Khan A, Nasir K, Khosa F, Saghir A, Sarwar S, Clouse ME. Prospective gating with 320MDCT angiography: effect of volume scan length on radiation dose. AJR Am J Roentgenol. 2011;196(2):407-11.

53. Vanhoenacker PK, Heijenbrok-Kal MH, Van Heste R, Decramer I, Van Hoe LR, Wijns W, et al. Diagnostic performance of multidetector CT angiography for assessment of coronary artery disease: meta-analysis. Radiology. 2007;244(2):419-28.

54. Miller JM, Rochitte CE, Dewey M, Arbab-Zadeh A, Niinuma H, Gottlieb I, et al. Diagnostic performance of coronary angiography by 64-row CT. N Engl J Med. 2008;359(22):2324-36.

55. Meijboom WB, Meijs MF, Schuijf JD, Cramer MJ, Mollet NR, van Mieghem CA, et al. Diagnostic accuracy of 64-slice computed tomography coronary angiography: a prospective, multicenter, multivendor study. J Am Coll Cardiol. 2008;52(25):2135-44.

56. Budoff MJ, Dowe D, Jollis JG, Gitter M, Sutherland J, Halamert E, et al. Diagnostic performance of 64-multidetector row coronary computed tomographic angiography for evaluation of coronary artery stenosis in individuals without known coronary artery disease: results from the prospective multicenter ACCURACY (Assessment by Coronary Computed Tomographic Angiography of Individuals Undergoing Invasive Coronary Angiography) trial. J Am Coll Cardiol. 2008;52(21):1724-32.

57. Achenbach S, Moselewski F, Ropers D, Ferencik M, Hoffmann U, MacNeill B, et al. Detection of calcified and noncalcified coronary atherosclerotic plaque by contrast-enhanced, submillimeter multidetector spiral computed tomography: a segment-based comparison with intravascular ultrasound. Circulation. 2004;109(1):14-7.

58. Leber AW, Knez A, Becker A, Becker C, von Ziegler F, Nikolaou K, et al. Accuracy of multidetector spiral computed tomography in identifying and differentiating the composition of coronary atherosclerotic plaques: a comparative study with intracoronary ultrasound. J Am Coll Cardiol. 2004;43(7):1241-7.

59. Leber AW, Knez A, von Ziegler F, Becker A, Nikolaou K, Paul S, et al. Quantification of obstructive and nonobstructive coronary lesions by 64-slice computed tomography: a comparative study with quantitative coronary angiography and intravascular ultrasound. J Am Coll Cardiol. 2005;46(1):147-54.

60. Leber AW, Becker A, Knez A, von Ziegler F, Sirol M, Nikolaou K, et al. Accuracy of 64-slice computed tomography to classify and quantify plaque volumes in the proximal coronary system: a comparative study using intravascular ultrasound. J Am Coll Cardiol. 2006;47(3):672-7. 
61. Motoyama S, Sarai M, Harigaya H, Anno H, Inoue K, Hara T, et al. Computed tomographic angiography characteristics of atherosclerotic plaques subsequently resulting in acute coronary syndrome. J Am Coll Cardiol. 2009;54(1):49-57.

62. Soeda T, Uemura S, Morikawa Y, Ishigami K, Okayama S, Hee SJ, et al. Diagnostic accuracy of dual-source computed tomography in the characterization of coronary atherosclerotic plaques: comparison with intravascular optical coherence tomography. Int $\mathrm{J}$ Cardiol. 2011;148(3):313-8.

63. Kashiwagi M, Tanaka A, Kitabata H, Tsujioka H, Kataiwa H, Komukai K, et al. Feasibility of noninvasive assessment of thin-cap fibroatheroma by multidetector computed tomography. JACC Cardiovasc Imaging. 2009;2(12):1412-9.

64. Maurovich-Horvat $\mathrm{P}$, Schlett CL, Alkadhi H, Nakano M, Stolzmann P, Vorpahl M, et al. Differentiation of early from advanced coronary atherosclerotic lesions: systematic comparison of CT, intravascular US, and optical frequency domain imaging with histopathologic examination in ex vivo human hearts. Radiology. 2012;265(2):393-401.

65. Wieringa WG, Lexis CP, Lipsic E, van der Werf HW, Burgerhof JG, Hagens VE, et al. In vivo coronary lesion differentiation with computed tomography angiography and intravascular ultrasound as compared to optical coherence tomography. J Cardiovasc Comput Tomogr. 2017;11(2):111-8.

66. Tomizawa N, Yamamoto K, Inoh S, Nojo T, Nakamura S. Accuracy of computed tomography angiography to identify thin-cap fibroatheroma detected by optical coherence tomography. J Cardiovasc Comput Tomogr. 2017;11(2):129-34.

67. Yang DH, Kang SJ, Koo HJ, Chang M, Kang JW, Lim TH, et al. Coronary CT angiography characteristics of OCT-defined thin-cap fibroatheroma: a section-to-section comparison study. Eur Radiol. 2018;28(2):833-43.

68. Miller JM, Dewey M, Vavere AL, Rochitte CE, Niinuma H, Arbab-Zadeh A, et al. Coronary CT angiography using 64 detector rows: methods and design of the multi-centre trial CORE- 64 . Eur Radiol. 2009;19(4):816-28.

69. Tenekecioglu E, Albuquerque FN, Sotomi Y, Zeng Y, Suwannasom P, Tateishi H, et al. Intracoronary optical coherence tomography: Clinical and research applications and intravascular imaging software overview. Catheter Cardiovasc Interv. 2017;89(4):679-89.

70. Cury RC, Abbara S, Achenbach S, Agatston A, Berman DS, Budoff MJ, et al. Coronary Artery Disease - Reporting and Data System (CAD-RADS): an Expert Consensus Document of SCCT, ACR and NASCI: endorsed by the ACC. JACC Cardiovasc Imaging. 2016;9(9):1099113. 
71. Petranovic M, Soni A, Bezzera H, Loureiro R, Sarwar A, Raffel C, et al. Assessment of nonstenotic coronary lesions by 64-slice multidetector computed tomography in comparison to intravascular ultrasound: evaluation of nonculprit coronary lesions. J Cardiovasc Comput Tomogr. 2009;3(1):24-31.

72. Arbab-Zadeh A, Hoe J. Quantification of coronary arterial stenoses by multidetector CT angiography in comparison with conventional angiography methods, caveats, and implications. JACC Cardiovasc Imaging. 2011;4(2):191-202.

73. Kashiwagi M, Tanaka A, Kitabata H, Ozaki Y, Komukai K, Tanimoto T, et al. Comparison of diagnostic accuracy between multidetector computed tomography and virtual histology intravascular ultrasound for detecting optical coherence tomography-derived fibroatheroma. Cardiovasc Interv Ther. 2014;29(2):102-8.

74. Ozaki Y, Okumura M, Ismail TF, Motoyama S, Naruse H, Hattori K, et al. Coronary CT angiographic characteristics of culprit lesions in acute coronary syndromes not related to plaque rupture as defined by optical coherence tomography and angioscopy. Eur Heart J.

2011;32(22):2814-23.

75. Ferencik M, Mayrhofer T, Puchner SB, Lu MT, Maurovich-Horvat P, Liu T, et al. Computed tomography-based high-risk coronary plaque score to predict acute coronary syndrome among patients with acute chest pain--Results from the ROMICAT II trial. J Cardiovasc Comput Tomogr. 2015;9(6):538-45.

76. Yamamoto H, Kitagawa T, Ohashi N, Utsunomiya H, Kunita E, Oka T, et al. Noncalcified atherosclerotic lesions with vulnerable characteristics detected by coronary CT angiography and future coronary events. J Cardiovasc Comput Tomogr. 2013;7(3):192-9.

77. Vancraeynest D, Pasquet A, Roelants V, Gerber BL, Vanoverschelde JL. Imaging the vulnerable plaque. J Am Coll Cardiol. 2011;57(20):1961-79. 


\begin{abstract}
Introduction: Multi-detector computed tomography is a non-invasive method for characterizing coronary atherosclerotic plaques. To date, no study has evaluated the ability of the 320-detector computed tomography to determine the various types of plaque. Objective: To assess the diagnostic accuracy of the 320-detector computed tomography in differentiating atherosclerotic plaques identified using the optical coherence tomography. Methods: We performed a retrospective, single center crosssectional study including 32 patients to compare findings of coronary atherosclerotic plaques using the 320-detector computed tomography and the optical coherence tomography in 42 coronary segments. The tomographic images were interpreted based on the new CAD-RADS classification. The 320-detector computed tomography images were independently evaluated by two radiologists and those of optical coherence tomography by an independent Corelab. Results: We observed an association between the findings of the atherosclerotic plaques in the optical coherence tomography and in the 320-detector computed tomography when results of these two methods were classified as calcified and non-calcified by the 320-detector computed tomography $(p=0.005)$. The 320-detector computed tomography was not able to identify fibroatheroma ( $p=0.49)$. Agreements of diameters (0.11 to 0.20$)$ and luminal area (0.52) measurements between the two methods were low. The 320-detector computed tomography underestimated luminal diameter values. The combination of napkin-ring sign, positive remodeling, low attenuation plaque and spotty calcification provided the highest accuracy $(75.14 \%$, 95\% confidence interval $60.8,89.5)$ to identify the fibroatheroma. The presence of at least two of these characteristics was associated with the presence of macrophage and cholesterol crystals observed by optical coherence tomography $(74.2 \%$ versus $25.8 \%, p=0.034$ and $85.7 \%$ versus $14.3 \%$; $p=0.040$, respectively). There was a trend towards a higher presence of napkin-ring sign $(80.0 \%$ versus $43.8 \%, p=0.071)$ in fibroatheromas observed by the optical coherence tomography. Conclusion: The 320-detector computed tomography was able to differentiate calcified plaques from non-calcified ones identified by the optical coherence tomography, but it could not correctly identify optical coherence tomography defined in fibroatheromas.
\end{abstract}

